Article

\title{
Implementation of Lightweight Convolutional Neural Networks via Layer-Wise Differentiable Compression
}

\author{
Huabin Diao ${ }^{1,2} \oplus$, Yuexing Hao ${ }^{1}$, Shaoyun $\mathrm{Xu}^{1, *}$ and Gongyan $\mathrm{Li}^{1}$ \\ 1 Institute of Microelectronics, Chinese Academy of Sciences, Beijing 100029, China; \\ diaohuabin@ime.ac.cn (H.D.); haoyuexing@ime.ac.cn (Y.H.); ligongyan@ime.ac.cn (G.L.) \\ 2 University of Chinese Academy of Sciences, Beijing 100049, China \\ * Correspondence: xushaoyun@ime.ac.cn
}

Citation: Diao, H.; Hao, Y.; Xu, S.; Li, G. Implementation of Lightweight Convolutional Neural Networks via Layer-Wise Differentiable Compression. Sensors 2021, 21, 3464 https://doi.org/10.3390/s21103464

Academic Editor: Dimitrios Moshou

Received: 24 March 2021

Accepted: 14 May 2021

Published: 16 May 2021

Publisher's Note: MDPI stays neutral with regard to jurisdictional claims in published maps and institutional affiliations.

Copyright: (c) 2021 by the authors. Licensee MDPI, Basel, Switzerland. This article is an open access article distributed under the terms and conditions of the Creative Commons Attribution (CC BY) license (https:/ / creativecommons.org/licenses/by/ $4.0 /)$.

\begin{abstract}
Convolutional neural networks (CNNs) have achieved significant breakthroughs in various domains, such as natural language processing (NLP), and computer vision. However, performance improvement is often accompanied by large model size and computation costs, which make it not suitable for resource-constrained devices. Consequently, there is an urgent need to compress CNNs, so as to reduce model size and computation costs. This paper proposes a layer-wise differentiable compression (LWDC) algorithm for compressing CNNs structurally. A differentiable selection operator OS is embedded in the model to compress and train the model simultaneously by gradient descent in one go. Instead of pruning parameters from redundant operators by contrast to most of the existing methods, our method replaces the original bulky operators with more lightweight ones directly, which only needs to specify the set of lightweight operators and the regularization factor in advance, rather than the compression rate for each layer. The compressed model produced by our method is generic and does not need any special hardware/software support. Experimental results on CIFAR-10, CIFAR-100 and ImageNet have demonstrated the effectiveness of our method. LWDC obtains more significant compression than state-of-the-art methods in most cases, while having lower performance degradation. The impact of lightweight operators and regularization factor on the compression rate and accuracy also is evaluated.
\end{abstract}

Keywords: convolutional neural networks; structural compression; differentiable; layer-wise

\section{Introduction}

In recent years, great breakthroughs have been achieved in information retrieval, natural language processing and computer vision due to the performance improvement of CNNs. However, the structure of CNN also becomes more complex, which brings large burdens of storage and computation. This greatly limits their deployment on resource-constrained devices, such as field-programmable gate arrays (FPGAs), digital signal processors (DSPs), cell phones and other mobile devices. Therefore, it is essential to obtain lightweight networks. There is some recent research to get efficient models by designing compact architectures manually, such as SqueezeNet [1], MobileNet [2-4] and ShuffleNet [5,6]. Some specific application scenarios of light CNNs also have be proposed, such as Crowd Counting [7], Bone Metastasis Classification [8] and Wheat Head Detection [9]. In addition, searching lightweight architectures automatically by neural architecture search [10-14] has become a research trend recently. In contrast to the aforementioned method of designing compact architectures straightforwardly, compressing the existing CNNs can also derive lightweight models. Current compression algorithms mainly focus on pruning the structural units within the CNNs, including filters, channels and other structural units. These compression algorithms cannot break the limitations of the origin network and can only prune units limitedly under a fixed network structure. In addition, they cannot achieve end-to-end compressing. Such a process is mainly divided into three steps: firstly pre-training the $\mathrm{CNN}$, then removing redundant structural 
units according to certain criterion and finally re-training the pruned model iteratively. In order to address the aforementioned problems, we propose a new $\mathrm{CNN}$ compression algorithm from a novel perspective. Instead of removing units from those redundant convolutional operators, we propose to replace them with more light ones directly, which allows us to break the limitations of original architecture. Our compression algorithm requires initially specifying $\mathrm{N}$ lightweight convolutional operators and then using them to reconstruct an over-parameterized $\mathrm{CNN}$ with $\mathrm{N}$ branches on each layer from a given original CNN. Each branch is multiplied by a trainable mask (whose value can only be 0 or 1), and only one branch among all branches on each layer has a mask of 1 . The reconstructed CNN can be trained via gradient descent with a resource-constrained objective function. At the end of training, the branches whose mask is 0 are removed from each layer and thus the lightweight model is constructed by the remaining branches with a mask of 1 . Consequently, compact CNNs composed of lightweight operators are derived.

In conclusion, the proposed approach is a layer-wise differentiable compression algorithm. Our main contributions can be summarized as follows:

- The proposed approach addresses the compression problem of CNNs from a fresh perspective, which replaces the original bulky operators with lightweight ones directly instead of pruning units from original redundant operators.

- Most of the existing approaches [15-19] require specifying the compression rate for each layer or require a threshold that is used to determine which structural units to prune. Our proposed approach does not require any such input and can automatically search for the best lightweight operator in each layer to replace the original redundant operator, thereby reducing the number of hyperparameters.

- The proposed approach is end-to-end trainable, which can compress and train CNNs simultaneously using gradient descent in one go. We can obtain various compressed lightweight CNNs with different architectures, which also inspires the future design of CNNs.

The rest of this paper is structured as follows: Section 2 describes the related works in $\mathrm{CNN}$ compression. Section 3 presents the proposed methodology. Section 4 describes the experiment in this paper and analyzes the experimental results. In Section 5, the conclusions are given.

\section{Related Works}

In the early stages, compression of CNNs focuses on fine-grained trimming. Although fine-grained compression methods [19] can achieve high pruning rates, the resulting sparse matrices require specialized hardware and software support, making it difficult to obtain actual acceleration. Thereby, the current $\mathrm{CNN}$ compression methods mainly focus on coarse-grained trimming, and the pruned units including channels, filters and other structural units. This paper mainly concentrates on coarse-grained compression methods, which include the following categories:

Trimming according to certain criteria: The main process of such pruning algorithms [18,20-24] includes: firstly training a CNN as usual, then pruning units from the trained CNN according to some artificial criteria, finally fine-tuning the slimmed CNN. Li et al. [18] rank the filters based on their norm values in each layer, removing the ones with small norm values. Hu et al. [21] sort the filters according to the ratio of activation values of zero (APoZ) in the feature map, pruning out the ones with larger APoZ values. He et al. [24] argue that norm-based pruning of filters gives better compression results only when the norm deviation of the filter is sufficiently large, so they propose a trimming method based on the geometric median of the filter. Singh et al. [23] order the filters by introducing an auxiliary loss function and evaluating the sensitivity of filter with respect to the auxiliary loss function, pruning out those sensitive ones. The aforementioned methods not only require a pruning criterion designed manually but also need to specify compressing rate, which increases the complexity of algorithm. 
Sparse regularization: Sparse regularization algorithms [25-29] realize CNN compression by introducing parameter-related regularization terms in the loss function and thus controlling the number of parameters in the network. Yoon et al. [26] add $\mathrm{L}(1,2)$ norms to the parameters at each layer of the network to learn fewer but more useful features to achieve model slimming. Ye et al. [27,29] compress CNNs by introducing the ADMM algorithm to optimize the model under a given parameter constraint.

Low-rank decomposition: Low-rank decomposition algorithms [30-32] use a lowerrank set instead of the original set of parameters to approximate the CNN to achieve compression. Swaminathan et al. [31] argue that the low-rank decomposition of weight matrices should consider influence of both input as well as output neurons of a layer. They propose a sparse low rank (SLR) approach that sparsifies SVD matrices to obtain better compression rate by keeping lower rank for unimportant neurons. Ruan et al. [32] construct a compressed-aware block to minimize the rank of the weight matrix and identify the redundant channels automatically.

Automatic pruning algorithm: He et al. [33] use a reinforcement learning method for $\mathrm{CNN}$ compression, encoding the compression rate of each layer as the agent's action, rewarding the agent with validation accuracy, and training the agent so that it can automatically determine the best compression rate used in each layer. Zhu et al. [34] set the compression rate of the model automatically with a heuristic method, however, which needs to determine the target compression rate of the model. Liu et al. [35] combine Alternating Direction Method of Multipliers (ADMM) [36] with the simulated annealing algorithm to automatically prune the network.

Knowledge distillation: Knowledge distillation [37-44] uses a complex CNN model with a large number of parameters to train a network with a small number of parameters to obtain a lightweight network. Wu et al. [42] propose a multi-teacher knowledge distillation framework to compress CNN. Prakosa et al. [43] explore that knowledge distillation can be integrated to pruning methodologies to improve accuracy of the pruned model. Ahmed et al. [44] propose a framework that leverages knowledge distillation and customizable block-wise optimization to learn a lightweight $\mathrm{CNN}$ architecture.

Most of the existing compression approaches can only prune a few redundant structural units from the fixed structure of the original network, which results in a low compression rate. In addition, they require specifying how many structural units from each layer to prune, which generates a lot of hyperparameters. In addition, the compression process requires pruning and retraining iteratively, which cannot be done in one go.

\section{Methodology}

\subsection{Overview}

The compression algorithm this paper proposed consists of three stages: the reconstructing stage, the searching stage and the fine-tuning stage. In the reconstructing stage, $\mathrm{N}$ lightweight convolutional operators are used to reconstruct an over-parameterized CNN with $\mathrm{N}$ branches on each layer from any given original $\mathrm{CNN}$. Each branch multiplies a trainable mask (whose value can only be 0 or 1 ) and only one branch among all branches on each layer has a mask of 1. The construction of the mask is described in Section 3.3.2. In the searching stage, the reconstructed $\mathrm{CNN}$ is trained via gradient descent with a resource-constrained objective function introduced in Section 3.3.3. At the end of training, the branches whose mask is 0 are removed from each layer in this reconstructed $\mathrm{CNN}$, thus a lightweight $\mathrm{CNN}$ is constructed by the remaining branches with a mask of 1 . In the fine-tuning stage, the lightweight $\mathrm{CNN}$ is fine-tuned to obtain a performance improvement.

\subsection{The Reconstructing Stage}

In the reconstructing stage, an over-parameterized CNN with multiple branches on each layer is reconstructed from any given original CNN. The lightweight CNN can be obtained by training this reconstructed CNN. Figure 1 shows one convolutional layer (left) in the original CNN and its corresponding layer (right) in the reconstructed CNN. 
Each convolutional layer (or pooling layer) is expanded into $\mathrm{N}$ parallel branches in the corresponding layer of the reconstructed $\mathrm{CNN}$.

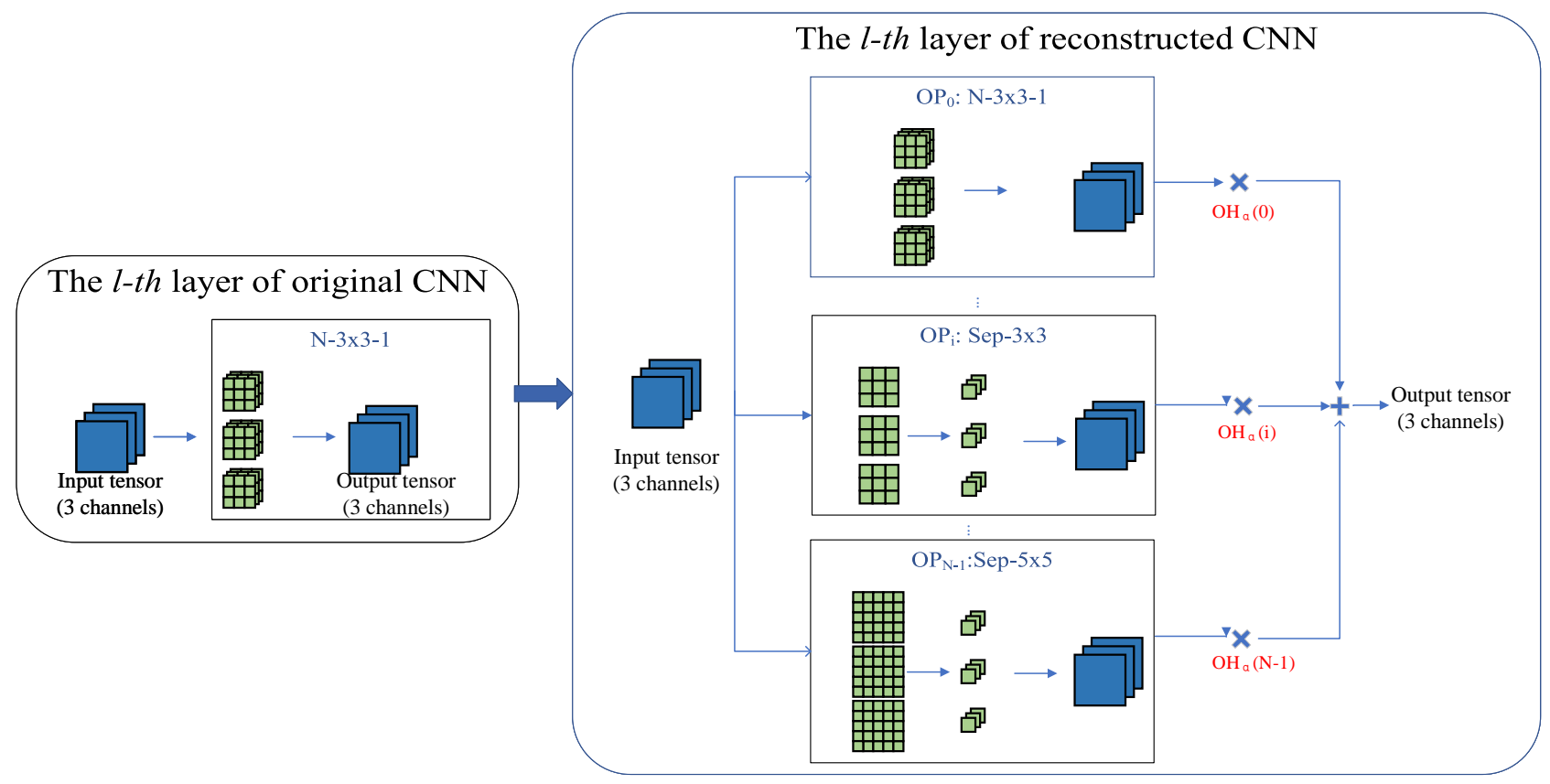

Figure 1. Reconstruction of the original CNN. We expand each layer (convolution layer or pooling layer) into $\mathrm{N}$ parallel branches and multiply a trainable mask to each branch. In this figure, $O H_{\alpha}$ is a one-hot mask vector generated by $\alpha$. Each element of $O H_{\alpha}$ can only be 0 or 1 and only one element can be $1 . O H_{\alpha}$ can be trained using gradient descend. $\alpha$ is defined as architecture parameter. The layer index of $\alpha$ is omitted which should be $\boldsymbol{\alpha}^{l}$ for the lth layer. The blue squares represent input or output tensors (with 3 channels for better presentation) and the green squares represent weights of convolution layers. Only three operators are presented in the figure, and more lightweight operators are shown in Appendix A.

The operators chosen in the branches are lightweight that have fewer parameters and floating-point operations (FLOPs) than the corresponding operators in the original CNN, such as group convolution [5,6], depthwise separable convolution [2,3] and CReLU convolution [37] (replacing ReLU with CReLU can save half of the channels). We experiment not only with these simple lightweight operators but also with other more complex lightweight convolutional modules, such as the Fire module [1], the module with residual connections [45]. We can also combine these different features to form new lightweight operators, e.g., combining the group feature with the $\mathrm{CReLU}$, or the depthwise separable feature. More details are shown in Appendix A, including the detailed structure, the number of parameters, and the FLOPs of these lightweight operators.

The output tensors from multiple branches are integrated into the final output tensor of this layer in the reconstructed CNN. We use weighted sum as the integration strategy and the weights are represented as $\mathrm{OH}_{\alpha}(\cdot)$ in Figure $1 . \mathrm{OH}_{\alpha^{l}}$ is a one-hot mask vector generated by $\boldsymbol{\alpha}^{l}$, and its construction is described in detail in Section 3.3.2. $l$ is the layer index. The convolutional operator for each branch is denoted as $O P_{i}, \mathrm{i}=0,1, \ldots, \mathrm{N}-1$. For ease of description, we define the convolutional operator corresponding to the reconstructed layer containing multiple branches as the selection operator $O S$, and then obtain

$$
O S^{l}(\cdot)=\sum_{i=0}^{N-1} O H_{\alpha^{l}}(i) \cdot O P_{i}(\cdot)
$$

\subsection{The Searching Stage}

In this section, a trainable gate function is constructed firstly, then a continuous approximation for the discrete one-hot mask vector $\mathrm{OH}_{\alpha}$ is performed based on the gate function. Next, the resource-constrained objective function is described and the reconstructed $\mathrm{CNN}$ 
is trained using it. In the searching stage, the value of mask vector $\mathrm{OH}_{\alpha}$ is simultaneously learned with the parameters of the convolutional operators of each layer. Additionally, there is only one branch per layer with a mask of 1 , and only the branch with a mask of 1 works, as shown in Figure 2.

\subsubsection{Trainable Gate Function}

The gate function $T G(\omega)$ is defined as

$$
\mathrm{TG}(\omega)= \begin{cases}1 & \omega>0 \\ 0 & \omega \leq 0\end{cases}
$$

The derivative of $T G(\omega)$ at any point except for the $\omega=0$ is 0 , so the function is not suitable for the gradient optimization process. It is necessary to approximate the gradient of $T G(\omega)$ so that it can be used for gradient descent. Kim et al. [46] directly use 1 to approximate the gradient of $T G(\omega)$, which called identity approximation. It can be observed from Figure $3 a$ that such approximation is very rough, which brings a large error due to the gradient mismatch. We introduce an asymptotic approximation function, denoted $A(\omega)$, which is inspired by the Error Decay Estimator (EDE) method proposed in [47], namely,

$$
\mathrm{TG}(\omega) \approx \mathrm{A}(\omega)=\mathrm{k} \cdot(\tanh (\mathrm{t} \cdot \omega)+1)
$$

where $\mathrm{t}=\mathrm{T}_{\min } 10^{\frac{i}{N} \times \log \left(\frac{T \max }{T \min }\right)}, \mathrm{k}=\frac{1}{2} \max \left(\frac{1}{t}, 1\right)$, and $\mathrm{T}_{\min }=0.1, T_{\max }=10$. In addition, $N$ denotes all the epochs required for training, $i$ represents the current epoch, $k$ and $t$ control the variation of $A(\omega)$ during the training process. Thus, the gradient of $T G(\omega)$ can be approximated as

$$
\frac{\partial T G(\omega)}{\partial \omega} \approx \frac{\partial \mathrm{A}(\omega)}{\partial \omega}=\mathrm{k} \cdot \mathrm{t} \cdot\left(1-\tanh (t \cdot w)^{2}\right)
$$

The l-th layer of reconstructed CNN

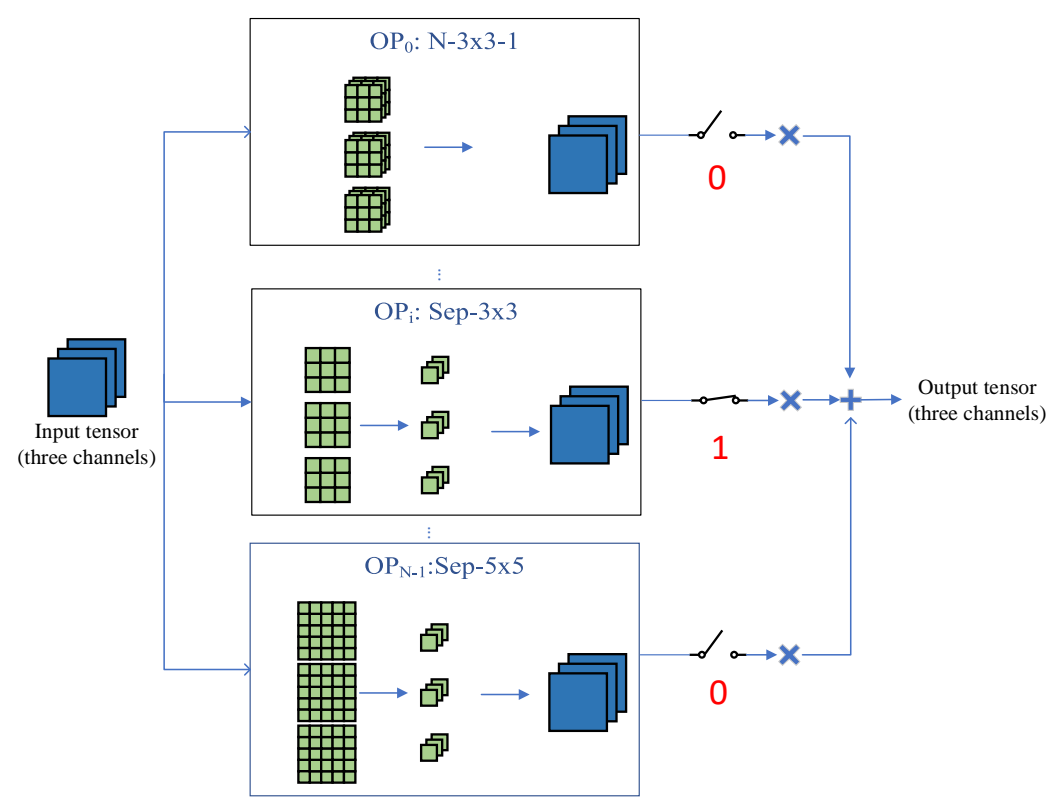

Figure 2. Illustration of the searching stage. In the searching stage, the mask of each branch $O H_{\alpha^{l}}(i)$ can be simultaneously learned with the parameters of convolutional operators. If $O H_{\boldsymbol{\alpha}^{l}}(i)=0$ in the current training step, it means the $i$ th branch does not work at this moment, and vice versa. The reconstructed $\mathrm{CNN}$ is equivalent to the child $\mathrm{CNN}$ formed by the branches whose mask is 1 in each training step. 


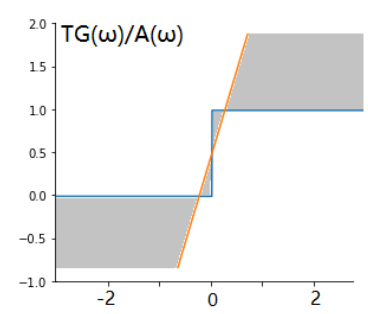

(a)

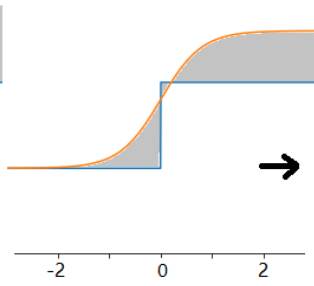

(b)

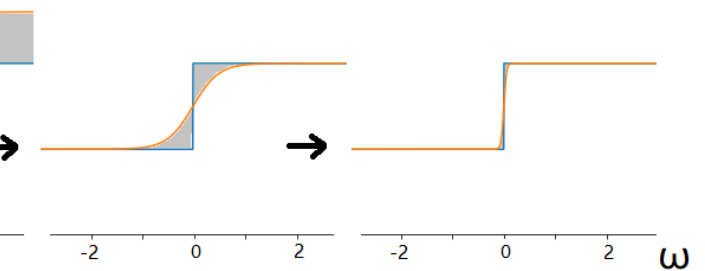

(c)

(d)

Figure 3. Illustration of the asymptotic approximation function. (a) The comparison between the identity approximation and the original function $T G(\omega)$, where the gray shaded part indicates the approximation error, indicating a large error in the identity approximation. (b-d) Different stages of asymptotic approximation, which gradually approximates $T G(\omega)$ as training proceeds. In the early stage of training, as shown in (b), the approximation error is large, but the updated parameters have a wide range; in the middle and later stages of training, as shown in $(\mathbf{c}, \mathbf{d})$, the approximation error gradually decreases, and the updated parameters are gradually concentrated around zero.

In conclusion, we construct a gate function that can be trained using gradient descent. By approximating the gate function $T G(\omega)$ asymptotically, the error can be reduced without sacrificing the ability of updating parameters.

\subsubsection{Continuous Approximation for Discrete One-Hot Vector}

In this section, a continuous one-hot mask vector $O H_{\alpha^{l}}$ ( $l$ is the layer index) is constructed based on $T G(\omega)$. The convolutional operator for each branch is defined as $O P_{i}$ and the length of $\mathrm{OH}_{\alpha^{l}}$ is equal to the number of branches. To construct $O H_{\alpha^{l}}$, additional $\left\lceil\log _{2} N\right\rceil$ parameters $(\lceil\cdot\rceil$ means upward rounding) need to be introduced, which are named architecture parameters $\alpha^{l}$ and expressed as $\left[\alpha_{0}^{l}, \alpha_{1}^{l}, \ldots, \alpha_{\left\lceil\log _{2} N\right\rceil-1}^{l}\right]$. Then, $O H_{\alpha^{l}}$ can be constructed using Equations (5) and (6), where $O H_{\alpha^{l}}(i)$ represents the $i$ th element of $O H_{\alpha^{l}}$.

$$
\begin{aligned}
& O H_{\boldsymbol{\alpha}^{l}}(i)=\prod_{j=0}^{\left\lceil\log _{2} N\right\rceil-1}\left(B_{j} \cdot T G\left(\boldsymbol{\alpha}_{j}^{l}\right)+\left(1-B_{j}\right) \cdot\left(1-T G\left(\boldsymbol{\alpha}_{j}^{l}\right)\right)\right)
\end{aligned}
$$

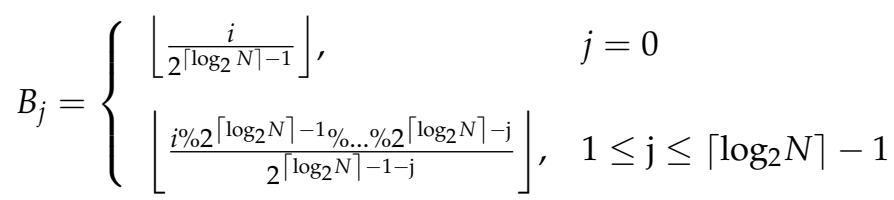

In the above equation, $\lfloor\cdot\rfloor, \%$ and $\prod$ represent downward rounding, remainder operation and multiplication operation, respectively. Next, the gradient of the architecture parameters $\alpha^{l}$ will be analyzed. Supposing the input tensor of the $l$ th layer is $\mathbf{x}^{l}$ and the output tensor is $\mathbf{y}^{l}$, which can be expressed as $\mathbf{y}^{l}=O S^{l}\left(\mathbf{x}^{l}\right)=\sum_{i=0}^{N-1} O H_{\boldsymbol{\alpha}^{l}}(i) \cdot O P_{i}\left(\mathbf{x}^{l}\right)$

Then, the gradient of $\alpha_{j}^{l}$ is

$$
\frac{\partial \mathbf{y}^{l}}{\partial \alpha_{j}^{l}}=\sum_{i=0}^{N-1} \frac{\partial O H_{\alpha^{l}}(i)}{\partial \alpha_{j}^{l}} \cdot O P_{i}\left(\mathbf{x}^{l}\right), j \in\left\{0,1, \ldots,\left\lceil\log _{2} N\right\rceil-1\right\}
$$

where

$$
\frac{\partial O H_{\alpha^{l}}(i)}{\partial \alpha_{j}^{l}}=\left(2 B_{j}-1\right) \cdot \frac{\partial T G\left(\alpha_{j}^{l}\right)}{\partial \alpha_{j}^{l}} \prod_{g=0, g \neq j}^{\left\lceil\log _{2} N\right\rceil-1}\left(B_{g} \cdot T G\left(\alpha_{g}^{l}\right)+\left(1-B_{g}\right) \cdot\left(1-T G\left(\alpha_{g}^{l}\right)\right)\right) .
$$

In the backpropagation process, $\alpha^{l}$ can be updated using the gradient obtained above. In conclusion, we construct a continuous one-hot mask vector $O H_{\alpha^{l}}$ using the architecture parameters $\alpha^{l}$ and derive the gradient of $\alpha^{l}$ to update $\alpha^{l}$ and $O H_{\alpha}$. 


\subsubsection{Resource-Constrained Objective Function}

The objective function is described as

$$
\arg \min _{\theta, \boldsymbol{\alpha}} L(\theta, \boldsymbol{\alpha})+\lambda \cdot R(\boldsymbol{\alpha})
$$

$\theta$ and $\alpha$ indicate the parameters of convolutional operators and architecture parameters, respectively. $L(\theta, \boldsymbol{\alpha})$ is the cross-entropy loss function used to measure the classification error of the model during the training process. $R(\boldsymbol{\alpha})$ is a regularization term used to measure the number of model parameters and FLOPs, which is only related to $\alpha . \lambda$ is the corresponding regularization factor.

In the following, we compute the regularization term $R(\boldsymbol{\alpha})$ for the reconstructed CNN. For the ith operator $O P_{i}$ in the $l$ th layer, the number of parameters and FLOPS can be represented as $P S_{i}^{l}$ and $F P_{i}^{l}$. Detailed equations are shown in Appendix A. Then, the total number of parameters and FLOPS of lth layer can be expressed as $P S^{l}=\sum_{i=0}^{N-1} O H_{\alpha^{l}}(i)$. $P S_{i}^{l}$ and $F P^{l}=\sum_{i=0}^{N-1} O H_{\alpha^{l}}(i) \cdot F P_{i}^{l}$, respectively. So far, the corresponding regularization term $R^{l}\left(\boldsymbol{\alpha}^{l}\right)$ of the lth layer can be denoted as $R^{l}\left(\boldsymbol{\alpha}^{l}\right)=\frac{1}{2}\left(\log \left(P S^{l}\right)+\log \left(F P^{l}\right)\right)$. The total regularization term $R(\boldsymbol{\alpha})$ is equal to the sum of $R^{l}\left(\boldsymbol{\alpha}^{l}\right)$ over all layers, and $\mathrm{L}$ is the number of CNN layers, we can obtain $R(\boldsymbol{\alpha})=\sum_{l=1}^{L} R^{l}\left(\boldsymbol{\alpha}^{l}\right)$.

\subsection{The Fine-Tuning Stage}

When the training in the searching stage is completed, all the branches with masks of 0 in the reconstructed $\mathrm{CNN}$ will be removed, only leaving the branch with a mask of 1 in each layer, as shown in Figure 4. Such behavior does not degrade the model performance, because those branches do not work. However, due to the potential problem of inadequate training in the searching stage, we perform the fine-tuning process on the pruned $\mathrm{CNN}$ to further improve performance. After the fine-tuning stage, the lightweight $\mathrm{CNN}$ is obtained.

\section{The l-th layer of compressed CNN}

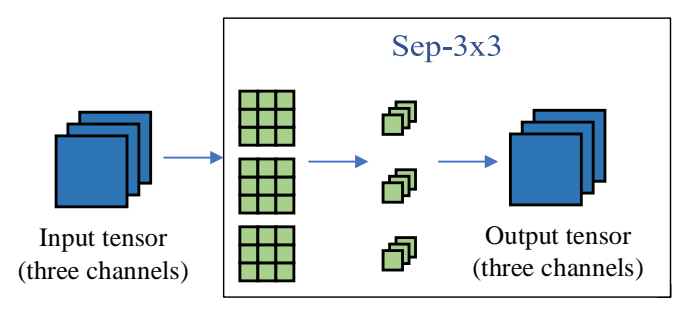

Figure 4. Illustration of the fine-tuning stage. Leaving only the branch with a mask of 1 in each layer and fine-tuning the compressed CNN for several epochs.

\section{Experiments}

\subsection{Dataset}

Cifar-10 [48]: The dataset has 60,000 images, each of which is an RGB three-channel image with a size of $32 \times 32$. It has 10 categories, with 6000 images per category. The dataset is divided into a training set and a validation set containing 50,000 and 10,000 images, respectively.

Cifar-100 [48]: The dataset also has 60,000 images, each of which is an RGB threechannel image with a size of $32 \times 32$. It has 100 categories with 600 images per category. The dataset is also divided into a training set and a validation set containing 50,000 and 10,000 images, respectively.

ImageNet-160-120 [49,50]: The dataset is built from ImageNet $16 \times 16$ [49], which is a down-sampled variant of ImageNet. The spatial resolution of ImageNet $16 \times 16$ is $16 \times 16$, and ImageNet-160-120 is constructed by selecting all images with label $\in[1,120]$ from 
ImageNet $16 \times 16$. Chrabaszcz [49] has proved that down-sampling images in ImageNet can significantly reduce computation costs for solving the optimal hyper-parameters of some classical models while maintaining similar search results. In summary, ImageNet160-120 contains $151.7 \mathrm{~K}$ training images and $6 \mathrm{~K}$ testing images with 120 classes.

\subsection{Evaluation Metrics}

We use the compression rate and TOP1 accuracy as evaluation metrics. The parameters compression ratio (PCR) and FLOPs compression ratio (FCR) are used to measure compression degree of a CNN model. PCR indicates the ratio of the number of parameters in the original $\mathrm{CNN}$ to the number of parameters in the compressed one and FCR indicates the ratio of the FLOPs in the original CNN to the FLOPs in the compressed one. The larger the PCR is, the smaller the model size will be. The larger the FCR is, the faster the model can be computed.

The formula for PCR and FCR are:

$$
\begin{aligned}
& P C R=\frac{\text { Params }_{\text {original }}}{\text { Params }_{\text {compressed }}} \\
& F C R=\frac{F L O P s_{\text {original }}}{F L O P s_{\text {compressed }}}
\end{aligned}
$$

\subsection{Results on Cifar}

We compress ResNet20, ResNet56, VGG16 on Cifar-10 and ResNet18 on Cifar-100. For those $\mathrm{CNNs}$, we do not compress the first convolutional layer and the last fully connected layer. The compression metrics, such as PCR and FCR, are calculated over all layers except the first convolutional layer and the last fully connected layer. The number of channels per convolutional layer for those CNNs is shown in Table 1.

The parameters $\theta$ of convolutional operators are optimized using the SGD optimizer with momentum, where the initial learning rate is 0.1 , the momentum is 0.9 and the weight decay is $3 \times 10^{-4}$. The learning rate is set using the CosineAnnealingLR scheme in Pytorch [51]. The architecture parameters $\alpha$ are optimized using Adam optimizer with an initial learning rate of 0.01 and a weight decay of $10^{-3}$, with the learning rate decaying by a factor of 0.3 every 40 epochs. Batch size is 256 , and the total training epochs are 150 . The architecture parameter $\alpha$ was randomly initialized using a normal distribution with mean 0 and variance 0.01 . The parameters $\theta$ and $\alpha$ are jointly trained.

Table 1. The number of channels in ResNet20, ResNet56, VGG16 and ResNet18.

\begin{tabular}{lccc}
\hline \multicolumn{5}{c}{ ResNet20 } \\
\hline Conv & conv1-6 & conv7-12 & conv13-18 \\
Channel & 16 & 32 & 64 \\
\hline \multicolumn{5}{c}{ ResNet56 } \\
\hline Conv & conv1-18 & conv19-36 & $64-54$ \\
Channel & 16 & 32 & conv8-13 \\
\hline \multicolumn{5}{c}{ VGG16 } \\
\hline Conv & conv1-2 & conv3-4 & conv5-7 \\
Channel & 64 & 128 & 256 \\
\hline \multicolumn{5}{c}{ conv13-16 } \\
\hline Conv & conv1-4 & ResNet18 & 512 \\
\hline
\end{tabular}


ResNet20 on Cifar-10: In ResNet20, convolutional operators with strides 1 and 2 are reconstructed to selection operators with strides 1 and 2, respectively. Different SOPs are used in compression experiments, and detailed information about SOPs is shown in Appendix C. ResNet20 is already a compact CNN, so there are few compression experiments on it. Therefore, we compare it directly with the baseline results. We use SOP1 and SOP2 to perform compression experiments on ResNet20, and set $\lambda$ to 0 and $1.5^{-3}$ respectively. Simple operators are used in SOP1 and SOP2, and the meanings of the operators can be found in Appendix A. SOP2 is more lightweight than SOP1. In the case of the same $\lambda$, it can be seen that the lighter the SOP is, the higher the compression degree of CNN will be, but the more the accuracy will drop. When using the same set of operators, the larger the $\lambda$ is, the higher the compression degree and accuracy drop will be, as shown in Table 2. It is consistent with common sense. The MA column in Table 2 represents the architecture of the compressed lightweight CNN, which can be found in Figures 5-7.

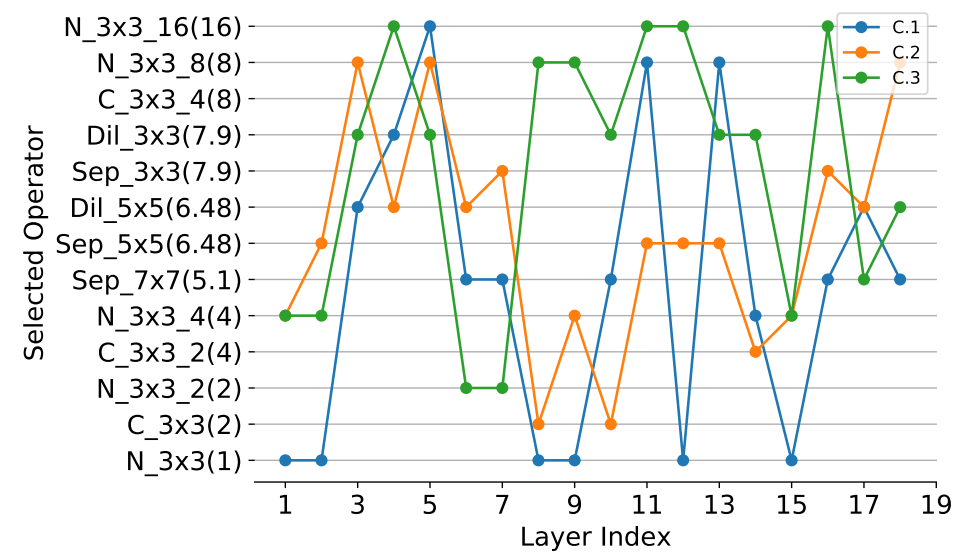

Figure 5. The operator of each layer in compressed ResNet20. The layers in the figure do not include the first convolutional layer and the last fully connected layer. From bottom to top on the vertical axis, the operators are increasingly lightweight. The float number in $(\cdot)$ represents the lightness of operator, the higher the value is, the lighter the operator is. The model located in the upper part of the figure is more lightweight.

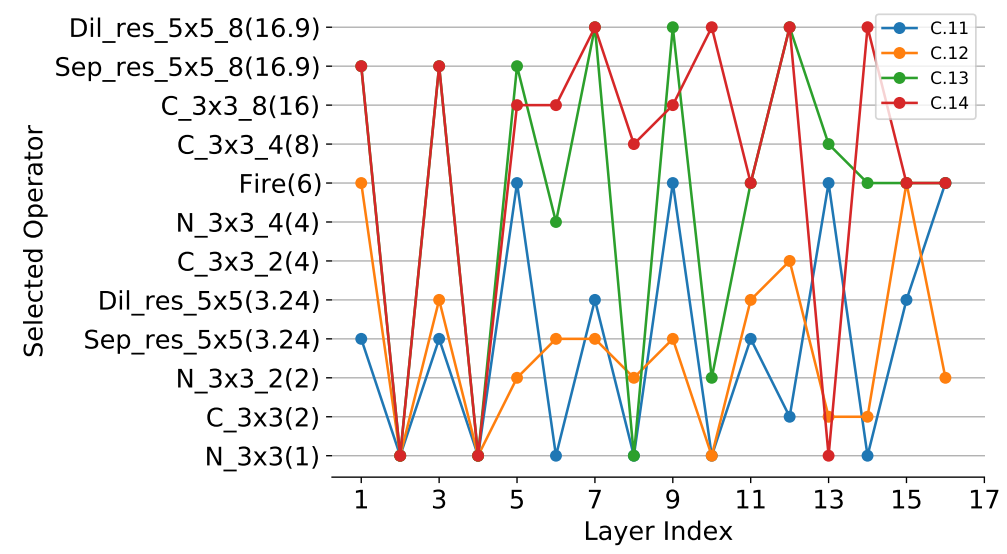

Figure 6. The operator of each layer in compressed ResNet18. The layers in the figure do not include the first convolutional layer and the last fully connected layer. The vertical axis has the same meanings as Figure 5. 
Table 2. Results of compressing ResNet20, ResNet56, VGG16 on Cifar-10 and ResNet18 on Cifar-100. SOP represents the set of operators used in the reconstructed CNN. TOP1 refers to the TOP1 accuracy of the model on the test set. MA is the architecture of the compressed model, as shown in Figures 5-7. Paras represents the number of parameters in the $\mathrm{CNN}$, containing the number of parameters of all convolutional layers except the first convolutional layer and the last fully connected layer.

\begin{tabular}{|c|c|c|c|c|c|c|c|c|}
\hline Model & Method & SOP & $\lambda$ & PCR & FCR & Paras & TOP1 (\%) & MA \\
\hline \multirow{4}{*}{ ResNet20 } & He. [45] & - & - & 0 & 0 & $0.27 \mathrm{M}$ & 91.25 & - \\
\hline & \multirow{3}{*}{ Ours } & SOP2 & 0 & 2.87 & 2.56 & $0.11 \mathrm{M}$ & 91.6 & C.1 \\
\hline & & SOP1 & $1.5 \times 10^{-3}$ & 4.91 & 4.19 & $0.06 \mathrm{M}$ & 90.35 & C. 2 \\
\hline & & SOP2 & $1.5 \times 10^{-3}$ & 6.48 & 5.61 & $0.047 \mathrm{M}$ & 90.15 & C. 3 \\
\hline \multirow{7}{*}{ ResNet56 } & He. [45] & - & - & 0 & 0 & $0.85 \mathrm{M}$ & 93.03 & - \\
\hline & Li. [18] & - & - & 1.16 & 1.38 & - & 93.06 & - \\
\hline & Dug. [52] & - & - & - & 2.12 & - & 92.72 & - \\
\hline & \multirow{4}{*}{ Ours } & SOP3 & 0 & 3.51 & 3.09 & $0.24 \mathrm{M}$ & 93.75 & C.7 \\
\hline & & SOP1 & 0 & 4.4 & 3.37 & $0.19 \mathrm{M}$ & 92.5 & C. 6 \\
\hline & & SOP1 & $1.5 \times 10^{-3}$ & 5.25 & 5.96 & $0.17 \mathrm{M}$ & 91.96 & C. 5 \\
\hline & & SOP2 & $1.5 \times 10^{-3}$ & 6.59 & 7.94 & $0.14 \mathrm{M}$ & 91.22 & C. 4 \\
\hline \multirow{6}{*}{ VGG16 } & Simon. [53] & - & - & 0 & 0 & $16.3 \mathrm{M}$ & 93.25 & - \\
\hline & Li [18] & - & - & 2.78 & 1.52 & - & 93.4 & - \\
\hline & Dug. [52] & - & - & 17.12 & 3.15 & - & 92.85 & - \\
\hline & \multirow{3}{*}{ Ours } & SOP5 & 0 & 2.82 & 3.71 & $5.79 \mathrm{M}$ & 94.65 & C.8 \\
\hline & & SOP4 & 0 & 3.85 & 2.61 & $4.23 \mathrm{M}$ & 93.95 & C.9 \\
\hline & & SOP6 & $1.5 \times 10^{-3}$ & 15.1 & 15.6 & $1.08 \mathrm{M}$ & 92.35 & C.10 \\
\hline \multirow{5}{*}{ ResNet18 } & He. [45] & & - & 0 & 0 & $11 \mathrm{M}$ & 75.05 & - \\
\hline & \multirow{4}{*}{ Ours } & SOP7 & 0 & 2.39 & 2.23 & $4.61 \mathrm{M}$ & 74.5 & C.11 \\
\hline & & SOP7 & $1.5 \times 10^{-3}$ & 2.44 & 2.31 & $4.5 \mathrm{M}$ & 74.2 & C.12 \\
\hline & & SOP8 & 0 & 5.27 & 2.97 & $2.08 \mathrm{M}$ & 74.85 & C.13 \\
\hline & & SOP8 & $1.5 \times 10^{-3}$ & 4.66 & 3.98 & $2.36 \mathrm{M}$ & 73.6 & C.14 \\
\hline
\end{tabular}

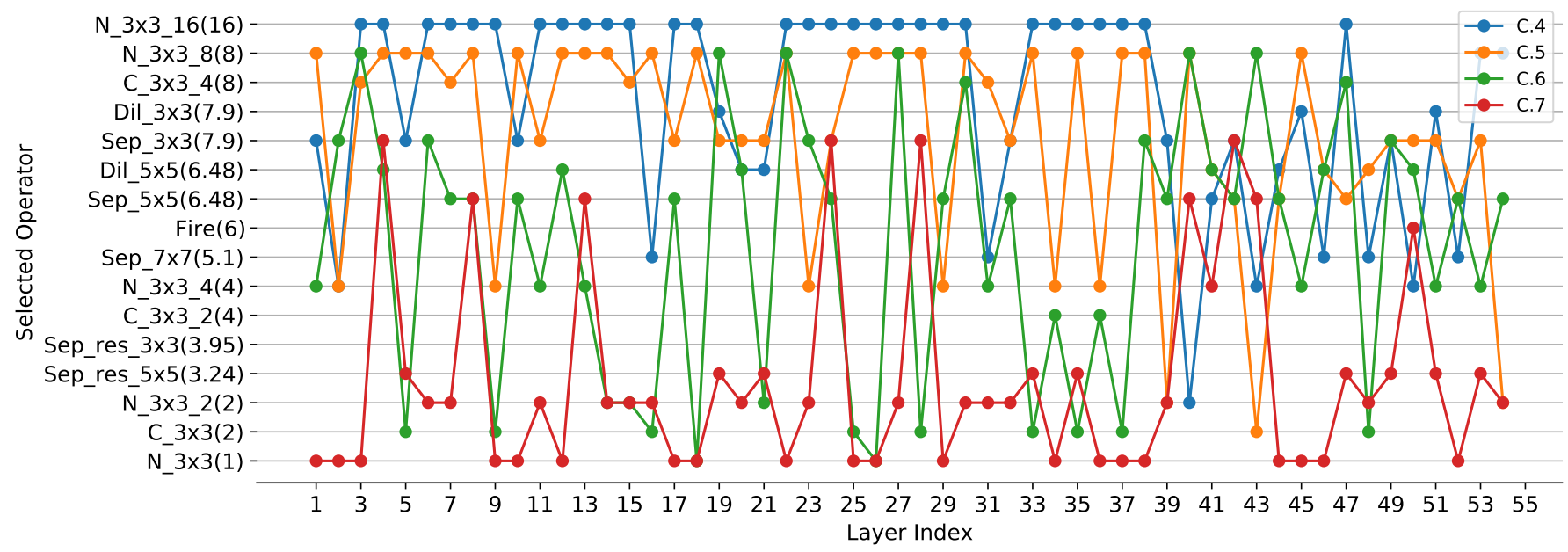

Figure 7. The operator of each layer in compressed ResNet56. The layers in the figure do not include the first convolutional layer and the last fully connected layer. The vertical axis has the same meanings as Figure 5.

ResNet56 on Cifar-10: The reconstruction of over-parameterized CNN for ResNet56 is similar to ResNet20. We use SOP1, SOP2 and SOP3 to perform compression experiments on ResNet56, and set $\lambda$ to 0 and $1.5^{-3}$, respectively. Complex operators have been added to SOP3, including the Fire module, the Sep_res_3 $\times 3$ module and the Sep_res_ $5 \times 5$ module. The meanings of these operators can be found in Appendix A. When using SOP1 with $\lambda$ set to $1.5^{-3}$, PCR and FCR of compressed model are 5.25 and 5.96, respectively, with TOP1 
accuracy being 91.96, as shown in Table 2. Keeping $\lambda$ constant, PCR and FCR increase to 6.59 and 7.94, respectively, when using a lighter SOP2, while TOP1 accuracy drops to 91.22. When using SOP1 with $\lambda$ reduced to 0, PCR and FCR decrease to 4.4 and 3.37 and TOP1 accuracy rises to 92.5, respectively. It can be seen that the larger the $\lambda$ is, the higher the compression degree of the model will be, but the more the accuracy will drop. When using SOP3 with complex operators, model accuracy of 93.75 can be achieved, indicating that the SOP is critical to the compression. For two operators of similar lightness, the complex operator is superior to the single operator.

VGG16 on Cifar-10: The convolutional operator in VGG16 is reconstructed to the selection operator with stride 1 and the pooling operator is reconstructed to the selection operator with stride 2. We use SOP4, SOP5 and SOP6 to perform compression experiments on VGG16. The meanings of the complex operators added in SOP4 and SOP5 can be found in Appendix A. SOP5 sets the group number of the group convolution in the complex operator to 1 , which has more parameters relative to the complex operator in SOP4. When using SOP5 with $\lambda$ set to 0 , the compressed model can achieve PCR and FCR of 2.82 and 3.71, respectively, with TOP1 accuracy being 94.65. To further improve the degree of compression, the $1 \times 1$ convolutional operator in the complex operators such as Sep_res_3 $\times$ 3, Sep_res_5 $\times 5$, Dil_res_3 $\times 3$ and Dil_res_5 $\times 5$ is modified to a group convolution operator with the group number of 4 to construct SOP4. As a result that these complex operators consist of depthwise separable convolution and $1 \times 1$ convolution operators, the $1 \times 1$ convolution operator plays a dominant role in the number of parameters and FLOPs in these complex operators when the number of convolution channels is large. When SOP4 is used to compress VGG16, PCR and FCR increase to 3.85 and 2.61, respectively, while TOP1 accuracy decreases to 93.95. PCR and FCR can even increase to 15.1 and 15.6 when using lighter SOP6, however, TOP1 accuracy drops to 92.35.

ResNet18 on Cifar-100: The reconstruction of over-parameterized CNN for ResNet18 is similar to ResNet20. We use SOP7 and SOP8 to perform compression experiments on ResNet18, and set $\lambda$ to 0 and $1.5^{-3}$, respectively. Compared to SOP7, SOP8 uses two more lightweight operators $C \_3 \times 3 \_4$ and $C \_3 \times 3 \_8$. The compressed model can achieve PCR and FCR of 2.39 and 2.23 by using SOP7 with $\lambda$ set to 0 and the TOP1 accuracy is 74.3, the obtained model architecture is shown in Figure 6.C.11. When increasing $\lambda$ to $1.5^{-3}$, PCR, FCR and TOP1 accuracy are 2.44, 2.34 and 74.2. To further improve the degree of compression, we use SOP8 and set $\lambda$ to 0 . The PCR and FCR of the compressed model are 5.27 and 2.97, and surprisingly the TOP1 accuracy is 74.85 . When $\lambda$ is set to $1.5^{-3}, \mathrm{PCR}$, FCR and TOP1 accuracy are 4.66, 3.98 and 73.6, as shown in Table 2.

\subsection{Results on ImageNet}

We compress DenseNet121 [54], MobileNetV2 [3] on ImageNet-16-120. Since the spatial resolution of ImageNet $16 \times 16$ is $16 \times 16$, we reserve only one downsampling layer with a stride 2 in these two models. In addition, we revise the classification layer from 1000D fully-connected to 120D fully-connected. We present their architectures in Tables 3 and 4, respectively. For DenseNet121, we only compress $3 \times 3$ convolutional operators in Dense Block. For MobileNetV2, we only compress those $1 \times 1$ convolutional operators in bottleneck. We do not compress the first convolutional layer and the last fully connected layer too. The experimental hyperparameters are the same with the experiments on Cifar, except that the batch size is modified to 512 . 
Table 3. DenseNet121 architectures for ImageNet-16-120.

\begin{tabular}{cccc}
\hline Layers & Output Size & Stride & Densenet121 \\
\hline conv & $16 \times 16$ & 1 & $3 \times 3$ conv \\
\hline Dense Block (1) & $16 \times 16$ & 1 & {$[3 \times 3$ conv $] \times 6$} \\
\hline Transition Layer (1) & $16 \times 16$ & 1 & $\begin{array}{c}1 \times 1 \text { conv } \\
2 \text { average pool }\end{array}$ \\
\hline Dense Block (2) & $16 \times 16$ & 1 & {$[3 \times 3$ conv $] \times 12$} \\
\hline Transition Layer (2) & $16 \times 16$ & 1 & $\begin{array}{c}1 \times 1 \text { conv } \\
2 \text { average pool }\end{array}$ \\
\hline Dense Block (3) & $16 \times 16$ & 1 & {$[3 \times 3$ conv $] \times 24$} \\
\hline Transition Layer (3) & $8 \times 8$ & 2 & $\begin{array}{c}1 \times 1 \text { conv } \\
2 \text { average pool }\end{array}$ \\
\hline Dense Block (4) & $8 \times 8$ & 1 & {$[3 \times 3$ conv $] \times 16$} \\
\hline $\begin{array}{c}\text { Classification } \\
\text { Layer }\end{array}$ & $1 \times 1$ & - & $8 \times 8$ average pool \\
\end{tabular}

DenseNet121 on ImageNet-16-120: In DenseNet121, those $3 \times 3$ convolutional operators in Dense Block are reconstructed to selection operators with stride 1 . We select the set of lightweight operators SOP9 for Densenet121 and then compress the model using different $\lambda$. The model size can be compressed by 3.42 times with a $0.23 \%$ decrease in accuracy when $\lambda$ is set to 0 . It is surprising that as $\lambda$ increases to $1.5 \times 10^{-4}$, not only does the compression rate increase to 5.13 , but the accuracy also increases by $0.13 \%$. As $\lambda$ keeps growing, the compression rate will continue to rise, along with more serious performance degradation. Furthermore, if $\lambda$ is smaller than $1.5 \times 10^{-3}$, a significant reduction in model size can be obtained by raising $\lambda$. However, once $\lambda$ exceeds $1.5 \times 10^{-3}$, the compression effect gained by increasing $\lambda$ will no longer be remarkable. More results are shown in Table 5.

MobileNetV2 on ImageNet-16-120: In MobileNetV2, we only compress those $1 \times 1$ convolutional operators in bottleneck, as the contribution of $3 \times 3$ depthwise separable convolution to model size is negligible. The lightweight operator set we used is SOP10, which consists of $1 \times 1$ group convolution, Fire, $3 \times 3$ group convolution and $3 \times 3$ CReLU group convolution. The detailed operators are given in the Appendix C. It is worth to notice that all the operators except the $1 \times 1$ group convolution in SOP10 have more parameters than the original $N \_1 \times 1$ convolution. For instance, the parameter volumes of Fire and $N \_3 \times 3 \_8$ are 1.5 and 1.12 times larger than $N \_1 \times 1$, respectively, with the same channel dimension. Hence, the model can achieve being compressed only when $\lambda$ is sufficiently large. As shown in Table 5, when $\lambda$ is 0 and $1.5 \times 10^{-4}$, although the accuracy of the obtained model is improved, the size is also larger than the original one. The model compression rate increases to 1.48 when $\lambda$ increases to $5 \times 10^{-4}$, and the accuracy also improves to 49.87 . Similarly, with increasing $\lambda$, there will be worse performance, although the model compression rate will continue to increase. As $\lambda$ proceeds to rise beyond $5 \times 10^{-3}$, the additional compression gain will be negligible. 
Table 4. MobileNetV2 architectures for ImageNet-16-120. In the table, bottleneck is $[1 \times 1$ conv, $3 \times 3 \mathrm{dw}$ - conv, $1 \times 1$ conv $]$, where $3 \times 3 \mathrm{dw}$-conv indicates $3 \times 3$ depthwise separable convolution. The meaning of Expansion Ratio can be seen from [3].

\begin{tabular}{cccccc}
\hline Layers & $\begin{array}{c}\text { Output } \\
\text { Size }\end{array}$ & Stride & Channels & $\begin{array}{c}\text { Expansion } \\
\text { Ratio }\end{array}$ & MobilenetV2 \\
\hline conv & $16 \times 16$ & 1 & 32 & - & $3 \times 3$ conv \\
\hline Block (1) & $16 \times 16$ & 1 & 16 & 1 & bottleneck $\times 1$ \\
\hline Block (2) & $16 \times 16$ & 1 & 24 & 6 & bottleneck $\times 2$ \\
\hline Block (3) & $16 \times 16$ & 1 & 32 & 6 & bottleneck $\times 3$ \\
\hline Block (4) & $16 \times 16$ & 1 & 64 & 6 & bottleneck $\times 4$ \\
\hline Block (5) & $16 \times 16$ & 1 & 96 & 6 & bottleneck $\times 3$ \\
\hline Block (6) & $8 \times 8$ & 2 & 160 & 6 & bottleneck $\times 3$ \\
\hline Block (7) & $8 \times 8$ & 1 & 320 & 6 & bottleneck $\times 1$ \\
\hline conv & $8 \times 8$ & 1 & 1280 & - & $1 \times 1$ conv \\
\hline Classification & $1 \times 1$ & - & - & - & $8 \times 8$ average pool \\
Layer & & & & & 120 Dfully-connected \\
\hline
\end{tabular}

Table 5. Results of compressing DenseNet121 and MobileNetV2 on ImageNet-16-120.

\begin{tabular}{|c|c|c|c|c|c|c|c|}
\hline Model & Method & SOP & $\lambda$ & PCR & Paras & TOP1 (\%) & $\overline{\text { MA }}$ \\
\hline \multirow{9}{*}{ DenseNet121 } & Huang. [54] & - & - & 0 & $9.84 \mathrm{M}$ & 48.83 & - \\
\hline & \multirow{8}{*}{ Ours } & SOP9 & 0 & 3.42 & $2.88 \mathrm{M}$ & 48.6 & C.15 \\
\hline & & SOP9 & $1.5 \times 10^{-4}$ & 5.13 & $1.92 \mathrm{M}$ & 48.73 & C.16 \\
\hline & & SOP9 & $5 \times 10^{-4}$ & 5.50 & $1.79 \mathrm{M}$ & 47.92 & C.17 \\
\hline & & SOP9 & $1.5 \times 10^{-3}$ & 5.96 & $1.65 \mathrm{M}$ & 47.9 & C. 18 \\
\hline & & SOP9 & $5 \times 10^{-3}$ & 6.0 & $1.64 \mathrm{M}$ & 47.83 & C.19 \\
\hline & & SOP9 & $1.5 \times 10^{-2}$ & 6.09 & $1.617 \mathrm{M}$ & 47.67 & C. 20 \\
\hline & & SOP9 & $5 \times 10^{-2}$ & 6.31 & $1.56 \mathrm{M}$ & 47.5 & C.21 \\
\hline & & SOP9 & $1.5 \times 10^{-1}$ & 6.47 & $1.52 \mathrm{M}$ & 47.2 & C. 22 \\
\hline \multirow{9}{*}{ MobileNetV2 } & sandler. [3] & - & - & 0 & $2.21 \mathrm{M}$ & 49.2 & - \\
\hline & \multirow{8}{*}{ Ours } & SOP10 & 0 & 0.45 & $4.89 \mathrm{M}$ & 49.3 & C. 23 \\
\hline & & SOP10 & $1.5 \times 10^{-4}$ & 0.71 & $3.12 \mathrm{M}$ & 49.4 & C. 24 \\
\hline & & SOP10 & $5 \times 10^{-4}$ & 1.48 & $1.49 \mathrm{M}$ & 49.87 & C. 25 \\
\hline & & SOP10 & $1.5 \times 10^{-3}$ & 2.48 & $0.89 \mathrm{M}$ & 49.06 & C. 26 \\
\hline & & SOP10 & $5 \times 10^{-3}$ & 2.91 & $0.76 \mathrm{M}$ & 48.95 & C. 27 \\
\hline & & SOP10 & $1.5 \times 10^{-2}$ & 2.83 & $0.78 \mathrm{M}$ & 48.96 & C. 28 \\
\hline & & SOP10 & $5 \times 10^{-2}$ & 3.05 & $0.725 \mathrm{M}$ & 48.75 & C. 29 \\
\hline & & SOP10 & $1.5 \times 10^{-1}$ & 3.06 & $0.723 \mathrm{M}$ & 48.68 & C. 30 \\
\hline
\end{tabular}

\subsection{Ablation Study}

Operation selection analysis: To evaluate the effectiveness of different lightweight operators in our method, experiments using different SOPs are performed without changing $\lambda$, and the results are shown in Tables 2 and 5 . We can find that using the lighter SOP yields a more compact model, for example, C. 6 with SOP1 located above C.7 with SOP3 in Figure 7 since SOP1 is lighter than SOP3. The same conclusion can be derived from Figures 5 and 6 . In addition, to study the performance of different operators, we perform multiple experiments with the same SOPs and different $\lambda$. It can be visualized from Figure 8 that $N \_3 \times 3 \_8$ is used most frequently in the compressed model, indicating that it is more effective, followed by Sep_3 $\times 3$ and Sep $\_5 \times 5$. In addition, $N \_3 \times 3 \_g$ has the 
same lightness compared with $C \_3 \times 3 \_g_{2}\left(g_{1}=2 g_{2}\right)$, however, $N \_3 \times 3 \_g_{1}$ is more likely to be selected during training, suggesting that $N_{-} 3 \times 3 \_g_{1}$ is more effective. Compared with simple operators, complex operators are more effective, such as Fire [1], Sep_res_5 $\times 5$ and Dil_res_5 × 5, as shown in Figure 9 .

From Figure 6 to Figure 7, it is interesting to see that the operators with higher lightness (e.g., $N \_3 \times 3 \_16$ ) tend to appear in the shallower layers (close to the input of models), while the operators with lower lightness (e.g., $N \_3 \times 3$ ) tend to appear in the deeper layers (close to the output of models). In our opinion, it is mainly due to the fact that shallow feature maps have higher resolution which leads to more redundant information, while deep feature maps have lower resolution and thus less redundant information. In addition, there is an alternation between operators of different lightness. The operators of higher lightness are often followed by several operators of lower lightness. We assume that appropriate redundancy is useful for training convergence. If the operators are too lightweight to extract sufficient information, it will tend to follow the less lightweight operators to regain more information, thus compensating for the model performance.

Effect of $\lambda$ : The compression rate is influenced by both SOP and $\lambda$. Once SOP has been selected, the maximum achievable compression rate is determined. As can be seen from Figure 10, the compression rate gradually grows as $\lambda$ increases, however, once $\lambda$ reaches a certain threshold, the improvement of compression rate will be insignificant. In this case, the compression rate is close to the maximum achievable value and the only choice to further raise the compression rate is to select a lighter SOP. Moreover, the threshold is related to the SOP, the lighter the SOP is, the smaller the threshold will be. From Figure 10, it is evident that the threshold is $1.5 \times 10^{-3}$ for Densenet121 and $5 \times 10^{-3}$ for MobileNetV2 since SOP9 is more lightweight than SOP10. $\lambda$ can prevent over-fitting, which is similar to what dropout does. It is clear that when $\lambda$ is relatively small, not only does the compression rate rise as $\lambda$ increases, but the accuracy of the model is also elevated. After $\lambda$ arrives at a critical value, the compression rate will continue to grow as $\lambda$ keeps increasing, but the model accuracy will begin to drop. Likewise, the critical value is also related to SOP, the lighter the SOP is, the smaller the critical value will be. The critical value of $\lambda$ is $1.5 \times 10^{-4}$ for Densenet121 and $5 \times 10^{-4}$ for MobileNetV2.

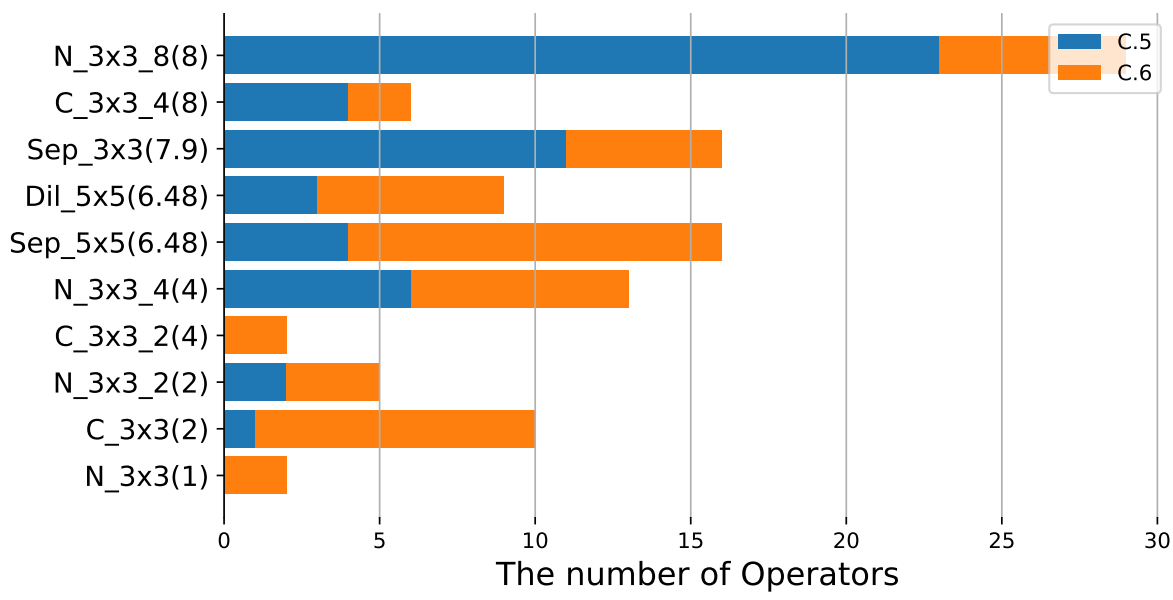

Figure 8. The distribution of operators in SOP1 when compressing resnet 56 on Cifar10. C.5 and C.6 correspond to $\lambda$ of $1.5^{-3}$ and 0 , respectively. $N \_3 \times 3 \_8$ appears the most times in this experiment, indicating that $N \_3 \times 3 \_8$ has a better effectiveness. As $\lambda$ increases, the algorithm tends to select the more lightweight operators, with blue histograms located in the upper part of the figure. 


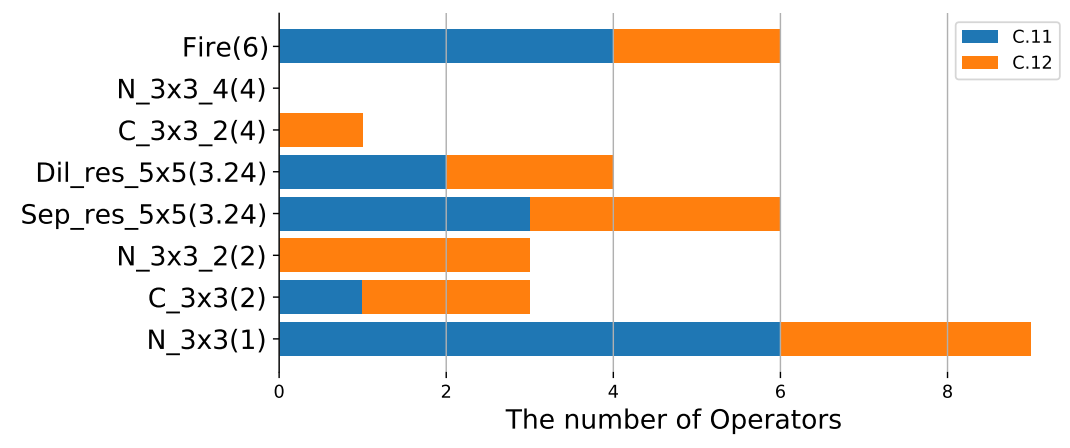

Figure 9. The distribution of operators in SOP7 when compressing resnet18 on Cifar100. C.11 and C.12 correspond to $\lambda$ of 0 and $1.5^{-3}$, respectively. Compared to simple operators, complex operators are more likely to be selected in compression training, indicating that complex operators are more effective, such as Fire, Sep_res_5 $\times 5$ and Dil_res_5 $\times 5$.

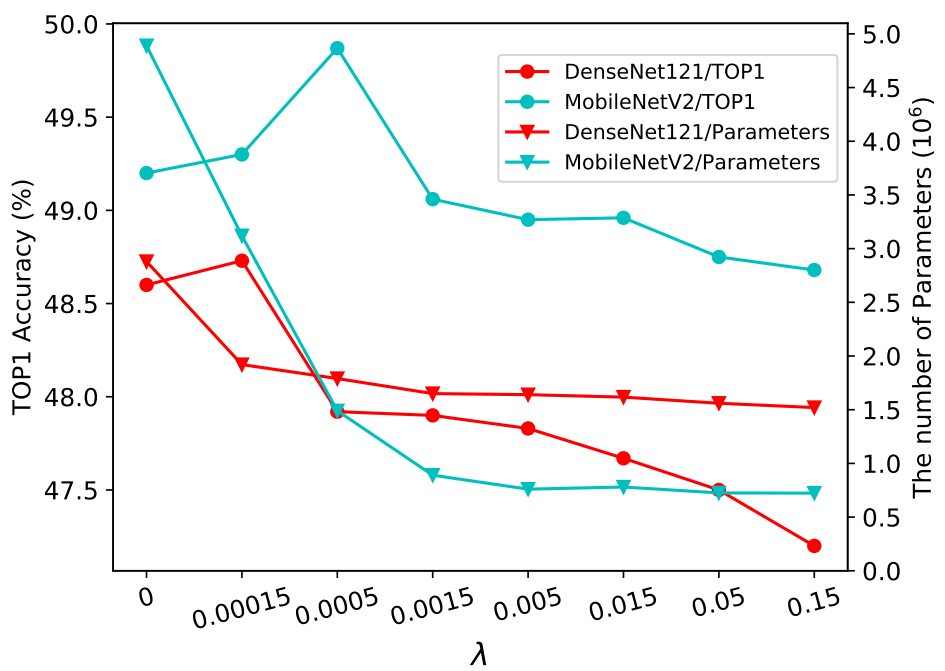

Figure 10. The effect of $\lambda$ on model size and TOP1 accuracy. For ease of visualization, the $\lambda$-axis is displayed with a uniform scale.

Algorithm extensibility: Our proposed differentiable selection operator is similar to common convolutional operator that can be trained using gradient descent directly. It not only can be embedded in a classification CNN, but also in a detection or segmentation CNN. Thus, our proposed compression algorithm is task-independent, and it not only can be applied to classification tasks but also to other vision tasks such as detection and segmentation. Furthermore, besides CNN, our proposed differentiable selection operator can be embedded in any networks that can be optimized by gradient descent, such as recurrent neural networks (RNN), generative adversarial networks (GAN), etc. From an intrinsic perspective, we propose a continuous approximation method for discrete one-hot vectors, which can be used not only for the model compression presented in this paper, but also for the network architecture search (NAS). In addition, the method can also be used for model quantization. If we use operators with different quantization bit widths to construct the selection operator, then we can obtain a mixed-precision quantization model after training.

Algorithm complexity: Selecting suitable operators manually to form a differentiable selection operator is the key point of our proposed compression algorithm. Fortunately, many lightweight operators are available for us. Once the selection operator is constructed, we can directly replace the original operator in the network to be compressed with it to construct an over-parameterized network. Then, we can train the network as we train a normal one. In addition, once the lightweight operators are selected, we only need to 
modulate $\lambda$ to achieve different levels of compression without setting the compression rate separately for each layer. Therefore, the algorithm is easy to implement. Compared to the original network, the over-parameterized network takes more time and memory to train, since each selection operator in it has $\mathrm{N}$ branches. Fortunately, we can alleviate the aforementioned problem. From the perspective of forward propagation, the output of selection operator is $\mathbf{y}=O S(\mathbf{x})=\sum_{i=0}^{N-1} O H_{\alpha}(i) \cdot O P_{i}(\mathbf{x})$. Since the mask vector $O H_{\alpha}$ is a one-hot vector, $\mathbf{y}$ is equal to the output of the branch $O P_{i}$ corresponding to $O H_{\alpha}(i)=1$, irrelevant to all other branches. From the perspective of back propagation, the update of architecture parameter $\alpha_{j}$ is only related to those branches with $\frac{\partial \mathrm{OH}(i)}{\partial \alpha_{j}} \neq 0$ according to Equation (7). Thus, we only need to compute those branches with $O H_{\alpha}(i)=1$ or $\frac{\partial O H_{\alpha}(i)}{\partial \alpha} \neq \mathbf{0}$ at each step. When there are 8 branches, only 4 branches are calculated on average at each step. This reduces memory consumption and training time significantly. For smaller models on Cifar, the compression process usually takes only 3-4 GPU hours, while for larger models on ImageNet, it usually takes 8-10 GPU hours.

\section{Conclusions}

A differentiable algorithm is proposed in this paper for CNN compression. Different from previous methods that prune redundant units from bulky convolutional operators, our method addresses the $\mathrm{CNN}$ compression problem from a completely new perspective by directly replacing the original bulky convolutional operators with more lightweight ones. The proposed approach is an end-to-end compression method that only requires control $\lambda$ to achieve different levels of compression, without specifying the compression rate for each layer. Specifically, our method can break the constraints of fixed operators in the network and obtain a higher compression rate without significant performance degradation. For example, the compressed ResNet20 and ResNet56 retain only $0.11 \mathrm{M}$ and $0.24 \mathrm{M}$ parameters, respectively, but their performance still outperforms the original network. A thorough comparison with several state-of-the-art compression methods proved the superiority of our proposed methodology on several highly competitive datasets. Overall, the proposed approach shows a unique potential for using gradient descent to seek the best lightweight operator for each layer to achieve compression, thus facilitating the application of CNNs on mobile and embedded devices.

Author Contributions: Conceptualization, H.D. and Y.H.; methodology, H.D.; software, S.X.; validation, H.D., Y.H. and S.X.; formal analysis, H.D.; investigation, H.D.; resources, G.L.; data curation, H.D.; writing—original draft preparation, H.D.; writing—review and editing, H.D.; visualization, Y.H.; supervision, G.L.; project administration, G.L.; funding acquisition, G.L. All authors have read and agreed to the published version of the manuscript.

Funding: This research was funded by National Key R\&D Program of China (No. 2018YFD0700300), and Chinese Academy of Sciences Engineering Laboratory for Intelligent Logistics Equipment System (No. KFJ-PTXM-025).

Institutional Review Board Statement: Not applicable.

Informed Consent Statement: Not applicable.

Data Availability Statement: Not applicable.

Conflicts of Interest: The authors declare no conflict of interest.

\section{Appendix A. Operators}

We experiment with different operators, and Table A1 lists the specific settings of the different operators and the corresponding number of parameters and FLOPs.

The description of simple symbols:

$C_{i}$ : Number of input channels; $C_{o}$ : Number of output channels; S: stride; P: padding; G: Number of groups; d: dilation; A: activation function; K: kernel size; PS: Number of parameters; Flops: floating-point operations; H,W: The height and width of the input tensor. 
N_3 $\times$ 3_g denotes a normal convolutional operation, where the number of groups $\mathrm{g}$ can be $1,2,4,8,16,32, \ldots$, and the group convolution is followed by the channel shuffle operation; $\mathbf{N} \_1 \times 1 \_$g is similar to $N_{-} 3 \times 3 \_g$, except that the kernel size is 1 ;

skip_connect denotes a shortcut that directly connects two nodes with the same input and output;

avg_pool_3 $\times 3$ denotes average pooling;

max_pool_3 $\times 3$ denotes maximum pooling;

Sep_k $\times \mathbf{k}$ denotes a depthwise separable convolution consisting of two convolutional layers, the first being a depthwise separable convolution with a convolutional kernel of $\mathrm{k}$ $\times \mathrm{k}$ and the second being a $1 \times 1$ convolution;

Dil_k $\times \mathbf{k}$ : denotes a dilated convolution consisting of two convolutional layers, the first of which is a depthwise separable dilated convolution with a convolutional kernel of $\mathrm{k} \times \mathrm{k}$ and the second of which is a $1 \times 1$ convolution;

C_3 $\times 3 \_g$ is similar to $N_{-} 3 \times 3 \_$, except that the activation function is replaced by CReLU from ReLU, where the number of groups can be $1,2,4,8,16,32, \ldots$, the group convolution is followed by the channel shuffle operation;

The description of complex symbols:

Fire: Using the Fire module in SqueezeNet, which consists of a squeeze layer with a convolutional kernel of $1 \times 1$ and two expand layers with a convolutional kernel of $1 \times 1$ and $3 \times 3$, respectively, where the number of input channels in the squeeze layer is $C_{i n}$ and the number of output channels is $C_{\text {squeeze }}$; the number of input channels in the expand layer is $C_{\text {squeeze }}$. The number of output channels is $\frac{C_{\text {out }}}{2}$, and the output of the Fire concatenates the outputs of the two expand layers together before outputting them. Here, we define $C_{\text {squeeze }}$ as $\min \left(\frac{C_{\text {out }}}{4}, 64\right)$;

Sep_res_k $\times$ k_g: Connect the two Sep_k $\times$ k convolutional operators using residuals, and that the $1 \times 1$ convolution layer in Sep_k $\times$ k uses group convolution with a group number of $g$, followed by the operation of channel shuffle;

Dil_res_k $\times$ k_g: Connect the two Dil_k $\times$ k convolutional operators using residuals, and that the $1 \times 1$ convolution layer in Dil_k $\times$ k uses group convolution with a group number of $g$, followed by the operation of channel shuffle.

Table A1. Different operators used in our experiments.

\begin{tabular}{|c|c|c|c|c|c|c|c|c|c|c|}
\hline Name & $C_{i}$ & $C_{o}$ & S & $\mathbf{P}$ & G & d & A & k & PS & Flops \\
\hline N_3 $\times 3 \_g$ & $C_{i}$ & $C_{o}$ & $S$ & 1 & $\mathrm{~g}$ & 1 & ReLU & $3 \times 3$ & $\frac{9 \cdot C_{i} \cdot C_{0}}{g}$ & $P S \cdot H \cdot W$ \\
\hline $\mathrm{N} \_1 \times 1 \_\mathrm{g}$ & $C_{i}$ & $C_{o}$ & S & 1 & $\mathrm{~g}$ & 1 & ReLU & $1 \times 1$ & $\frac{C_{i}^{8} \cdot C_{0}}{g}$ & $P S \cdot H \cdot W$ \\
\hline skip_connect & $C_{i}$ & $C_{i}$ & - & - & - & - & - & - & 0 & 0 \\
\hline avg_pool_3 $\times 3$ & $C_{i}$ & $C_{i}$ & $S$ & 1 & 1 & 1 & ReLU & $3 \times 3$ & 0 & $9 \cdot C_{i} \cdot H \cdot W$ \\
\hline max_pool_3 $\times 3$ & $C_{i}$ & $C_{i}$ & $S$ & 1 & 1 & 1 & ReLU & $3 \times 3$ & 0 & $9 \cdot C_{i} \cdot H \cdot W$ \\
\hline Sep_3 33 & $C_{i}$ & $C_{o}$ & $S$ & 1 & $C_{i}$ & 1 & ReLU & $3 \times 3$ & $9 \cdot C_{i}+C_{i} \cdot C_{o}$ & $P S \cdot H \cdot W$ \\
\hline Sep_5 $\times 5$ & $C_{i}$ & $C_{o}$ & $S$ & 2 & $C_{i}$ & 1 & ReLU & $5 \times 5$ & $25 \cdot C_{i}+C_{i} \cdot C_{o}$ & $P S \cdot H \cdot W$ \\
\hline Sep_7 $\times 7$ & $C_{i}$ & $C_{o}$ & S & 3 & $C_{i}$ & 1 & ReLU & $7 \times 7$ & $49 \cdot C_{i}+C_{i} \cdot C_{o}$ & $P S \cdot H \cdot W$ \\
\hline Dil_3 $\times 3$ & $C_{i}$ & $C_{0}$ & $S$ & 2 & $C_{i}$ & 2 & ReLU & $3 \times 3$ & $9 \cdot C_{i}+C_{i} \cdot C_{o}$ & $P S \cdot H \cdot W$ \\
\hline Dil_5 × 5 & $C_{i}$ & $C_{o}$ & $S$ & 4 & $C_{i}$ & 2 & ReLU & $5 \times 5$ & $25 \cdot C_{i}+C_{i} \cdot C_{o}$ & $P S \cdot H \cdot W$ \\
\hline C_3 $\times 3$ _g & $C_{i}$ & $\frac{C_{0}}{2}$ & S & 1 & $\mathrm{~g}$ & 1 & CReLU & $3 \times 3$ & $\frac{9 \cdot C_{i} \cdot C_{0}}{2 \cdot g}$ & $P S \cdot H \cdot W$ \\
\hline Fire & $C_{i}$ & $C_{0}$ & S & 1 & 1 & 1 & ReLU & $3 \times 3$ & $\frac{C_{i}^{2}}{4}+\frac{5 \cdot C_{i} \cdot C_{0}}{4}$ & $P S \cdot H \cdot W$ \\
\hline Sep_res_3 $\times 3$ _g & $C_{i}$ & $C_{o}$ & S & 1 & g & 1 & ReLU & $3 \times 3$ & $\frac{C_{i}^{2}+C_{i} \cdot C_{o}}{g}+18 \cdot C_{i}$ & $P S \cdot H \cdot W$ \\
\hline Sep_res_5 $\times 5$ _g & $C_{i}$ & $C_{o}$ & $S$ & 1 & $\mathrm{~g}$ & 1 & ReLU & $5 \times 5$ & 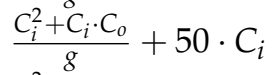 & $P S \cdot H \cdot W$ \\
\hline Dil_res_3 × 3_g & $C_{i}$ & $C_{o}$ & S & 2 & g & 2 & ReLU & $3 \times 3$ & $\frac{C_{i}^{2}+\dot{C}_{i} \cdot C_{o}}{g}+18 \cdot C_{i}$ & $P S \cdot H \cdot W$ \\
\hline Dil_res_5 $\times 5 \_g$ & $C_{i}$ & $C_{o}$ & $S$ & 1 & $\mathrm{~g}$ & 1 & ReLU & $5 \times 5$ & $\frac{C_{i}^{2}+C_{i} \cdot C_{0}}{g}+50 \cdot C_{i}$ & $P S \cdot H \cdot W$ \\
\hline
\end{tabular}




\section{Appendix B. More Detailed Experimental Results}

In this section, the lightweight architectures of VGG16, DenseNet121 and MobileNetV2 after being compressed are presented in detail.

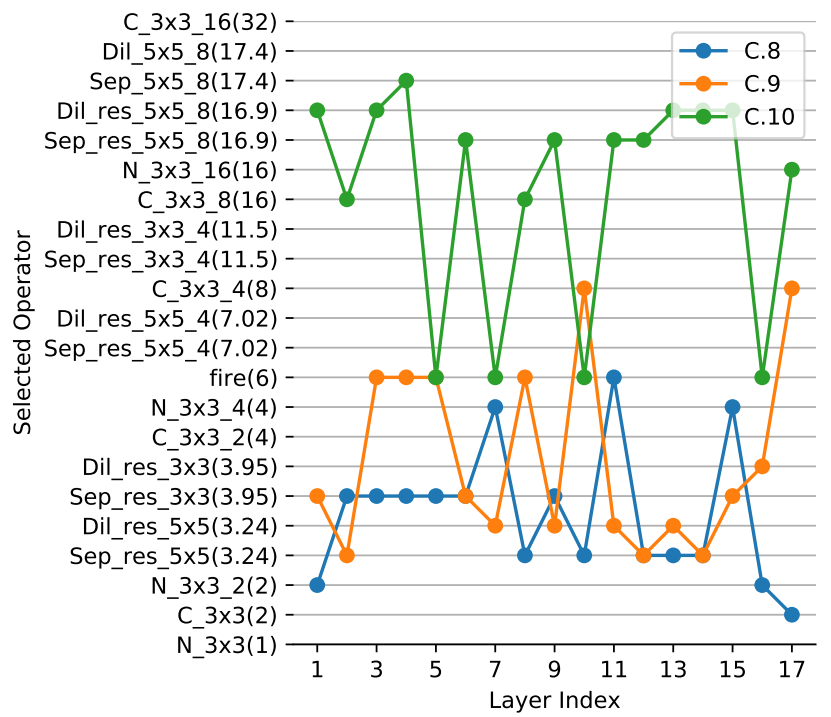

Figure A1. The operator of each layer in compressed VGG16. The layers in the figure do not include the first convolutional layer and the last fully connected layer. The vertical axis have the same meanings as Figure 5.

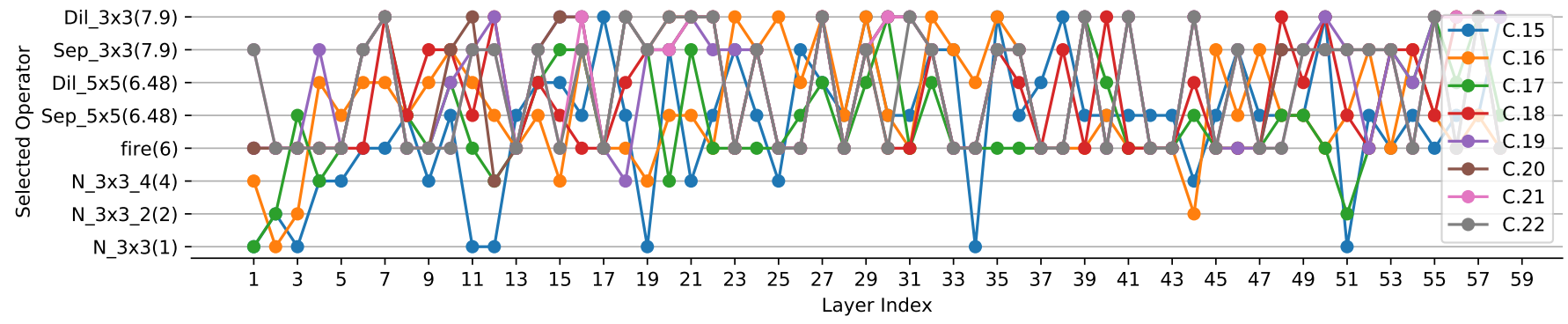

Figure A2. The operator of each layer in compressed DenseNet121. The layers in the figure do not include the first convolutional layer and the last fully connected layer. The vertical axis has the same meanings as Figure 5.

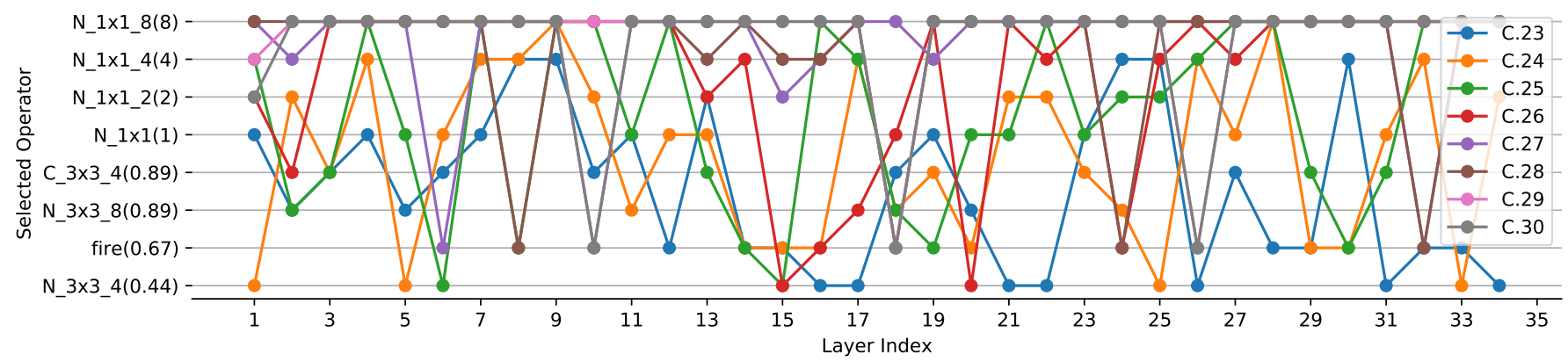

Figure A3. The operator of each layer in compressed MobileMetV2. The layers in the figure do not include the first convolutional layer and the last fully connected layer. The vertical axis has the same meanings as Figure 5. 


\section{Appendix C. Different Sets of Operators in the Branches of Reconstructed CNN (SOP)}

Table A2. Different sets of operators in the branches of reconstructed CNN.

\begin{tabular}{|c|c|c|c|c|}
\hline \multicolumn{5}{|c|}{ SOP1 } \\
\hline N_3 $\times 3$ & N_3 $\times 3 \_2$ & N_3 $\times 3 \_4$ & N_3 $\times 3 \_8$ & Sep_3 $\times 3$ \\
\hline C_3 $3 \times 3$ & C_3 $3 \times 3 \_2$ & C_3 $3 \times 3 \_4$ & Dil_5 $\times 5$ & Sep_5 $\times 5$ \\
\hline \multicolumn{5}{|c|}{ SOP2 } \\
\hline N_3 $\times 3$ & N_3 $\times 3 \_2$ & N_3 $\times 3 \_4$ & Sep_5 $\times 5$ & Dil_3 $\times 3$ \\
\hline N_3 $\times 3 \_8$ & N_3 $\times 3 \_16$ & Sep_3 33 & Sep_7x7 & Dil_5 55 \\
\hline \multicolumn{5}{|c|}{ SOP3 } \\
\hline N_3 $\times 3$ & N_3 $\times 3 \_2$ & N_3 $\times 3 \_4$ & Sep_res_3 33 & Sep_3 $\times 3$ \\
\hline Fire & C_3 $3 \times 3$ & C_3 $3 \times 3 \_2$ & Sep_res_5 $\times 5$ & Sep_5 $\times 5$ \\
\hline \multicolumn{5}{|c|}{ SOP4 } \\
\hline Fire & C_3 $3 \times 3 \_2$ & Sep_res_3 $\times 3 \_4$ & Sep_res_5 $\times 5 \_4$ & \\
\hline C_3 $3 \times 3 \_4$ & N_3 $\times 3$ & Dil_res_3 $\times$ 3_4 & Dil_res_5 $\times 5 \_4$ & \\
\hline \multicolumn{5}{|c|}{ SOP5 } \\
\hline N_3 $\times 3$ & N_3 $\times 3 \_2$ & N_3 $\times 3 \_4$ & Sep_res_3 $\times 3$ & Dil_res_3 $\times 3$ \\
\hline Fire & C_3 $3 \times 3$ & C_3 $3 \times 3 \_2$ & Sep_res_5 $\times 5$ & Dil_res_5 $\times 5$ \\
\hline \multicolumn{5}{|c|}{ SOP6 } \\
\hline Fire & C_3 $3 \times 3 \_16$ & Sep_res_5 $\times 5$ _8 & Sep_5 $\times 5 \_8$ & \\
\hline$\overline{C \_} 3 \times 3 \_8$ & N_3 $\times 3 \_16$ & Dil_res_5 $\times 5 \_8$ & Dil_5 $\times 5 \_8$ & \\
\hline \multicolumn{5}{|c|}{ SOP7 } \\
\hline N_3 $\times 3$ & N_3 $\times 3 \_2$ & N_3 $\times 3 \_4$ & Dil_res_5 5 5 & \\
\hline Fire & C_3 $3 \times 3$ & C_3 $3 \times 3 \_2$ & Sep_res_5 $\times 5$ & \\
\hline \multicolumn{5}{|c|}{ SOP8 } \\
\hline N_3 $\times 3$ & N_3 $\times 3 \_2$ & N_3 $\times 3 \_4$ & Dil_res_5 $\times 5 \_8$ & \\
\hline Fire & C_3 $3 \times 3 \_4$ & C_3 $3 \times 3 \_8$ & Sep_res_5 $\times 5$ _8 & \\
\hline \multicolumn{5}{|c|}{ SOP9 } \\
\hline N_3 $\times 3$ & N_3 $\times 3 \_2$ & N_3 $\times 3 \_4$ & Dil_3 33 & \\
\hline Fire & Dil_5 $\times 5$ & Sep_3 $\times 3$ & Sep_5 $\times 5$ & \\
\hline \multicolumn{5}{|c|}{ SOP10 } \\
\hline N_1 $1 \times 1$ & N_1 $1 \times 1 \_2$ & N_1 $1 \times 1 \_4$ & N_1 $1 \times 1 \_8$ & \\
\hline C_3 $3 \times 3 \_4$ & N_3 $\times 3 \_4$ & N_3 $\times 3 \_8$ & Fire & \\
\hline
\end{tabular}

\section{References}

1. Iandola, F.N.; Han, S.; Moskewicz, M.W.; Ashraf, K.; Dally, W.J.; Keutzer, K. Squeezenet: Alexnet-level accuracy with 50x fewer parameters and $<0.5 \mathrm{mb}$ model size. arXiv 2016, arXiv:1602.07360.

2. Howard, A.G.; Zhu, M.; Chen, B.; Kalenichenko, D.; Wang, W.; Weyand, T.; Andreetto, M.; Adam, H. Mobilenets: Efficient convolutional neural networks for mobile vision applications. arXiv 2017, arXiv:1704.04861.

3. Sandler, M.; Howard, A.; Zhu, M.; Zhmoginov, A.; Chen, L.C. Mobilenetv2: Inverted residuals and linear bottlenecks. In Proceedings of the IEEE Conference on Computer Vision and Pattern Recognition, Salt Lake City, UT, USA, 18-22 June 2018; pp. 4510-4520. 
4. Howard, A.; Sandler, M.; Chu, G.; Chen, L.C.; Chen, B.; Tan, M.; Wang, W.; Zhu, Y.; Pang, R.; Vasudevan, V.; et al. Searching for mobilenetv3. In Proceedings of the IEEE International Conference on Computer Vision, Seoul, Korea, 27-28 October 2019; pp. 1314-1324.

5. Ma, N.; Zhang, X.; Zheng, H.T.; Sun, J. Shufflenet v2: Practical guidelines for efficient cnn architecture design. In Proceedings of the European Conference on Computer Vision (ECCV), Munich, Germany, 8-14 September 2018; pp. 116-131.

6. Zhang, X.; Zhou, X.; Lin, M.; Sun, J. Shufflenet: An extremely efficient convolutional neural network for mobile devices. In Proceedings of the IEEE Conference on Computer Vision and Pattern Recognition, Salt Lake City, UT, USA, 18-23 June 2018; pp. 6848-6856.

7. Wang, P.; Gao, C.; Wang, Y.; Li, H.; Gao, Y. Mobilecount: An efficient encoder-decoder framework for real-time crowd counting. Neurocomputing 2020, 407, 292-299. [CrossRef]

8. Ntakolia, C.; Diamantis, D.E.; Papandrianos, N.; Moustakidis, S.; Papageorgiou, E.I. A lightweight convolutional neural network architecture applied for bone metastasis classification in nuclear medicine: A case study on prostate cancer patients. Healthcare 2020, 8, 493. [CrossRef]

9. Khaki, S.; Safaei, N.; Pham, H.; Wang, L. Wheatnet: A lightweight convolutional neural network for high-throughput image-based wheat head detection and counting. arXiv 2021, arXiv:2103.09408.

10. Liu, H.; Simonyan, K.; Yang, Y. Darts: Differentiable architecture search. arXiv 2018, arXiv:1806.09055.

11. Xie, S.; Zheng, H.; Liu, C.; Lin, L. Snas: Stochastic neural architecture search. arXiv 2018, arXiv:1812.09926.

12. Zoph, B.; Vasudevan, V.; Shlens, J.; Le, Q.V. Learning transferable architectures for scalable image recognition. In Proceedings of the IEEE Conference on Computer Vision and Pattern Recognition, Salt Lake City, UT, USA, 18-23 June 2018; pp. 8697-8710.

13. Dai, X.; Yin, H.; Jha, N.K. Nest: A neural network synthesis tool based on a grow-and-prune paradigm. IEEE Trans. Comput. 2019, 68, 1487-1497. [CrossRef]

14. Real, E.; Aggarwal, A.; Huang, Y.; Le, Q.V. Regularized evolution for image classifier architecture search. Proc. AAAI Conf. Artif. 2019, 33, 4780-4789. [CrossRef]

15. Ding, X.; Ding, G.; Han, J.; Tang, S. Auto-balanced filter pruning for efficient convolutional neural networks. AAAI $2018,3,7$.

16. He, Y.; Kang, G.; Dong, X.; Fu, Y.; Yang, Y. Soft filter pruning for accelerating deep convolutional neural networks. arXiv 2018, arXiv:1808.06866.

17. He, Y.; Zhang, X.; Sun, J. Channel pruning for accelerating very deep neural networks. In Proceedings of the IEEE International Conference on Computer Vision, Venice, Italy, 22-29 October 2017; pp. 1389-1397.

18. Li, H.; Kadav, A.; Durdanovic, I.; Samet, H.; Graf, H.P. Pruning filters for efficient convnets. arXiv 2016, arXiv:1608.08710.

19. Han, S.; Mao, H.; Dally, W.J. Deep compression: Compressing deep neural networks with pruning, trained quantization and huffman coding. arXiv 2015, arXiv:1510.00149.

20. Guo, Y.; Yao, A.; Chen, Y. Dynamic network surgery for efficient dnns. arXiv 2016, arXiv:1608.04493.

21. Hu, H.; Peng, R.; Tai, Y.W.; Tang, C.K. Network trimming: A data-driven neuron pruning approach towards efficient deep architectures. arXiv 2016, arXiv:1607.03250.

22. Luo, J.H.; Wu, J.; Lin, W. Thinet: A filter level pruning method for deep neural network compression. In Proceedings of the IEEE International Conference on Computer Vision, Venice, Italy, 22-29 October 2017; pp. 5058-5066.

23. Singh, P.; Kadi, V.S.; Verma, N.; Namboodiri, V.P. Stability based filter pruning for accelerating deep cnns. In Proceedings of the 2019 IEEE Winter Conference on Applications of Computer Vision (WACV), Waikoloa, HI, USA, 7-11 January 2019; pp. 1166-1174.

24. He, Y.; Liu, P.; Wang, Z.; Hu, Z.; Yang, Y. Filter pruning via geometric median for deep convolutional neural networks acceleration. In Proceedings of the IEEE Conference on Computer Vision and Pattern Recognition, Long Beach, CA, USA, 16-20 June 2019; pp. 4340-4349.

25. Wen, W.; Wu, C.; Wang, Y.; Chen, Y.; Li, H. Learning structured sparsity in deep neural networks. arXiv 2016, arXiv:1608.03665.

26. Yoon, J.; Hwang, S.J. Combined group and exclusive sparsity for deep neural networks. In Proceedings of the International Conference on Machine Learning, Sydney, Australia, 6-11 August 2017; pp. 3958-3966.

27. Zhang, T.; Ye, S.; Zhang, K.; Tang, J.; Wen, W.; Fardad, M.; Wang, Y. A systematic dnn weight pruning framework using alternating direction method of multipliers. In Proceedings of the European Conference on Computer Vision (ECCV), Munich, Germany, 8-14 September 2018; pp. 184-199.

28. Ma, Y.; Chen, R.; Li, W.; Shang, F.; Yu, W.; Cho, M.; Yu, B. A unified approximation framework for compressing and accelerating deep neural networks. In Proceedings of the IEEE 31st International Conference on Tools with Artificial Intelligence (ICTAI), Portland, OR, USA, 4-6 November 2019; pp. 376-383.

29. Ye, S.; Feng, X.; Zhang, T.; Ma, X.; Lin, S.; Li, Z.; Xu, K.; Wen, W.; Liu, S.; Tang, J.; et al. Progressive dnn compression: A key to achieve ultra-high weight pruning and quantization rates using admm. arXiv 2019, arXiv:1903.09769.

30. Gusak, J.; Kholiavchenko, M.; Ponomarev, E.; Markeeva, L.; Blagoveschensky, P.; Cichocki, A.; Oseledets, I. Automated multi-stage compression of neural networks. In Proceedings of the IEEE International Conference on Computer Vision Workshops, Seoul, Korea, 27-28 October 2019.

31. Swaminathan, S.; Garg, D.; Kannan, R.; Andres, F. Sparse low rank factorization for deep neural network compression. Neurocomputing 2020, 398, 185-196. [CrossRef]

32. Ruan, X.; Liu, Y.; Yuan, C.; Li, B.; Hu, W.; Li, Y.; Maybank, S. Edp: An efficient decomposition and pruning scheme for convolutional neural network compression. IEEE Trans. Neural Netw. Learn. Syst. 2020. [CrossRef] 
33. He, Y.; Lin, J.; Liu, Z.; Wang, H.; Li, L.J.; Han, S. Amc: Automl for model compression and acceleration on mobile devices. In Proceedings of the European Conference on Computer Vision (ECCV), Munich, Germany, 8-14 September 2018; pp. 784-800.

34. Zhu, M.; Gupta, S. To prune, or not to prune: Exploring the efficacy of pruning for model compression. arXiv 2017, arXiv:1710.01878.

35. Liu, N.; Ma, X.; Xu, Z.; Wang, Y.; Tang, J.; Ye, J. Autocompress: An automatic dnn structured pruning framework for ultra-high compression rates. AAAI 2020, 34, 4876-4883. [CrossRef]

36. Boyd, S.; Parikh, N.; Chu, E. Distributed Optimization and Statistical Learning via the Alternating Direction Method of Multipliers; Now Publishers Inc.: Delft, The Netherlands, 2011.

37. Shang, W.; Sohn, K.; Almeida, D.; Lee, H. Understanding and improving convolutional neural networks via concatenated rectified linear units. In Proceedings of the International Conference on Machine Learning, New York, NY, USA, 19-24 June 2016; pp. 2217-2225.

38. Bhardwaj, K.; Suda, N.; Marculescu, R. Dream distillation: A data-independent model compression framework. arXiv 2019, arXiv:1905.07072.

39. Koratana, A.; Kang, D.; Bailis, P.; Zaharia, M. Lit: Learned intermediate representation training for model compression. In Proceedings of the International Conference on Machine Learning, Long Beach, CA, USA, 10-15 June 2019; pp. 3509-3518.

40. Wang, J.; Bao, W.; Sun, L.; Zhu, X.; Cao, B.; Philip, S.Y. Private model compression via knowledge distillation. Proc. AAAI Conf. Artif. 2019, 33, 1190-1197. [CrossRef]

41. Wang, Z.; Lin, S.; Xie, J.; Lin, Y. Pruning blocks for cnn compression and acceleration via online ensemble distillation. IEEE Access 2019, 7, 175703-175716. [CrossRef]

42. Wu, M.C.; Chiu, C.T. Multi-teacher knowledge distillation for compressed video action recognition based on deep learning. J. Syst. Archit. 2020, 103, 101695. [CrossRef]

43. Prakosa, S.W.; Leu, J.S.; Chen, Z.H. Improving the accuracy of pruned network using knowledge distillation. Pattern Anal. Appl. 2020, 1-12. [CrossRef]

44. Ahmed, W.; Zunino, A.; Morerio, P.; Murino, V. Compact cnn structure learning by knowledge distillation. In Proceedings of the 2020 25th International Conference on Pattern Recognition (ICPR), Milan, Italy, 10-15 January 2021.

45. He, K.; Zhang, X.; Ren, S.; Sun, J. Deep residual learning for image recognition. In Proceedings of the IEEE conference on computer vision and pattern recognition, Las Vegas, NV, USA, 27-30 June 2016; pp. 770-778.

46. Kim, J.; Park, C.; Jung, H.J.; Choe, Y. Plug-in, trainable gate for streamlining arbitrary neural networks. AAAI 2020, 34, 4452-4459. [CrossRef]

47. Qin, H.; Gong, R.; Liu, X.; Shen, M.; Wei, Z.; Yu, F.; Song, J. Forward and backward information retention for accurate binary neural networks. In Proceedings of the IEEE/CVF Conference on Computer Vision and Pattern Recognition, Seattle, WA, USA, 13-19 June 2020; pp. 2250-2259.

48. Krizhevsky, A.; Hinton, G. Learning Multiple Layers of Features from Tiny Images; University of Toronto: Toronto, ON, Canada, 2009.

49. Chrabaszcz, P.; Loshchilov, I.; Hutter, F. A downsampled variant of imagenet as an alternative to the cifar datasets. arXiv 2017, arXiv:1707.08819

50. Dong, X.; Yang, Y. Nas-bench-201: Extending the scope of reproducible neural architecture search. arXiv 2020, arXiv:2001.00326.

51. Paszke, A.; Gross, S.; Massa, F.; Lerer, A.; Bradbury, J.; Chanan, G.; Killeen, T.; Lin, Z.; Gimelshein, N.; Antiga, L.; et al. Pytorch: An imperative style, high-performance deep learning library. In Advances in Neural Information Processing Systems 32; Wallach, H., Larochelle, H., Beygelzimer, A., d'Alché-Buc, F., Fox, E., Garnett, R., Eds.; Curran Associates, Inc.: Dutchess County, NY, USA, 2019; pp. 8024-8035.

52. Duggal, R.; Xiao, C.; Vuduc, R.; Sun, J. Cup: Cluster pruning for compressing deep neural networks. arXiv 2019, arXiv:1911.08630.

53. Simonyan, K.; Zisserman, A. Very deep convolutional networks for large-scale image recognition. arXiv 2014, arXiv:1409.1556.

54. Huang, G.; Liu, Z.; Van Der Maaten, L.; Weinberger, K.Q. Densely connected convolutional networks. In Proceedings of the 2017 IEEE Conference on Computer Vision and Pattern Recognition (CVPR), Honolulu, HI, USA, 21-26 July 2017; pp. 2261-2269. 